\title{
Survey of Deep Representation Learning for Speech Emotion Recognition
}

\author{
Siddique Latif, Rajib Rana, Sara Khalifa, Raja Jurdak, Senior Member, IEEE, Junaid Qadir, Senior \\ Member, IEEE, and Björn Schuller, Fellow, IEEE
}

\begin{abstract}
Traditionally, speech emotion recognition (SER) research has relied on manually handcrafted acoustic features using feature engineering. However, the design of handcrafted features for complex SER tasks requires significant manual effort, which impedes generalisability and slows the pace of innovation. This has motivated the adoption of representation learning techniques that can automatically learn an intermediate representation of the input signal without any manual feature engineering. Representation learning has led to improved SER performance and enabled rapid innovation. Its effectiveness has further increased with advances in deep learning $(\mathrm{DL})$, which has facilitated deep representation learning where hierarchical representations are automatically learned in a data-driven manner. This paper presents the first comprehensive survey on the important topic of deep representation learning for SER. We highlight various techniques, related challenges and identify important future areas of research. Our survey bridges the gap in the literature since existing surveys either focus on SER with hand-engineered features or representation learning in the general setting without focusing on SER.
\end{abstract}

Index Terms-Speech emotion recognition, multi task learning, representation learning, domain adaptation, unsupervised learning

\section{INTRODUCTION}

$S^{\mathrm{r}}$ Peech is a natural mode of communication among humans. It conveys affective information about emotional expression through explicit (linguistic) and implicit (paralinguistic) cues. Studies report that linguistic messages are rather unreliable means to predict and analyse human affective behaviour [1] because linguistic content is language-dependent, and the generalisation of emotions for multiple languages is very difficult to achieve. People often choose different words to express emotion, making it hard to anticipate a speaker's word choice and the associated affective expressions. The paralinguistic content of speech, on the other hand, provides an immense body of acoustic features that can be used to encode the emotional state of the speaker. These acoustic features are reliable indicators of basic emotions and have been explored by different machine learning (ML) [2]-[4] as well as deep learning (DL) models [5]-[8] for speech emotion recognition (SER).

Traditionally, the efficiency of ML algorithms in SER has been critically dependent on the quality of hand-crafted acoustic features. Consequently, feature engineering, which focuses on creating features from raw speech, has been an important part of SER research for a long time. Deep

- S. Latif is affiliated with University of Southern Queensland (USQ), Australia and Distributed Sensing Systems Group, Data61-CSIRO, Australia.

- R. Rana is with University of Southern Queensland (USQ), Australia.

- S. Khalifa is affiliated with Distributed Sensing Systems Group, Data61-CSIRO, University of New South Wales, and University of Queensland, Australia.

- R. Jurdak is with the Trusted Networks Lab, Queensland University of Technology (QUT), Australia.

- J. Qadir is affiliated with the Qatar University, Doha, Qatar and the Information Technology University (ITU), Lahore, Pakistan.

- B. Schuller is affiliated with GLAM - the Group on Language, Audio, and Music, Imperial College London, UK, and the ZD.B Chair of Embedded Intelligence for Health Care and Wellbeing, University of Augsburg, Germany.

Corresponding E-mail: siddique.latif@usq.edu.au

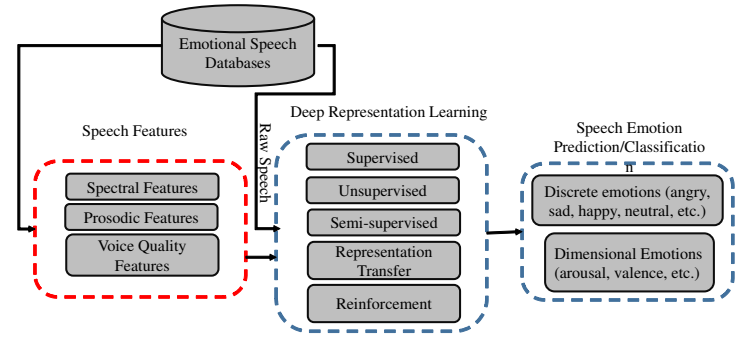

Fig. 1: An overview of deep representation learning-based SER system.

representation learning encompasses DL techniques to learn representations of input data, usually through the nonlinear transformation of the input data. Researchers have evaluated different DL models for representation learning in SER. Figure 1 presents an overview of deep representation learning in SER, showing that DL models can learn emotionally salient representations from raw speech as well as from acoustic features. These deep models can be trained in different ways, including supervised, unsupervised, semisupervised, and transfer learning techniques to learn emotional representation from speech. This review covers all these deep representation learning techniques for SER proposed to date.

We compare our paper with the existing surveys on deep learning or representation learning for SER in Table 1. The comparison shown demonstrates the uniqueness of our paper. The article by Bengio et al. [9] focus on the geometrical connections between representation learning, manifold learning, and density estimation. Since this article was published in 2013, it predated the development of modern generative models, and the discussions focus mostly on traditional techniques such as principal component analysis (PCA) [14], restricted Boltzmann machines (RBMs), and autoencoders (AEs). Research on representation learn- 
TABLE 1: Comparison of our paper with that of the existing surveys

\begin{tabular}{|c|c|c|c|c|c|c|}
\hline \multirow{3}{*}{ Paper } & \multicolumn{5}{|c|}{ Focus } & \multirow{3}{*}{ Details } \\
\hline & \multirow{2}{*}{$\begin{array}{l}\text { Representation } \\
\text { Learning }\end{array}$} & \multicolumn{3}{|c|}{ Speech Emotion Recognition } & \multirow{2}{*}{$\begin{array}{l}\text { Deep } \\
\text { Learning }\end{array}$} & \\
\hline & & Datasets & $\begin{array}{l}\text { Hand Engineered } \\
\text { Feature }\end{array}$ & $\begin{array}{l}\text { Deep Representation } \\
\text { Learning }\end{array}$ & & \\
\hline $\begin{array}{l}\text { Bengio et al. [9] } \\
2013\end{array}$ & $\checkmark$ & $x$ & ( & 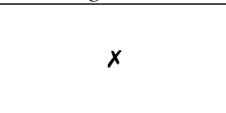 & $\checkmark$ & $\begin{array}{l}\text { This paper reviews the work in the area of unsupervised } \\
\text { feature learning and deep learning. It also covers } \\
\text { advancements in probabilistic models and autoencoders. } \\
\text { It does not include recent models like VAE and GANs. }\end{array}$ \\
\hline $\begin{array}{l}\text { Zhong et al. [10] } \\
2016\end{array}$ & $\checkmark$ & $x$ & $x$ & $x$ & $\checkmark$ & $\begin{array}{l}\text { This paper covers the history of data representation } \\
\text { learning from traditional to recent DL methods. Challenges } \\
\text { for deep representation learning, recent advancement, } \\
\text { and future directions are not covered. }\end{array}$ \\
\hline $\begin{array}{l}\text { Basu et al. [11] } \\
2017\end{array}$ & $x$ & $\checkmark$ & $\checkmark$ & $x$ & $\checkmark$ & $\begin{array}{l}\text { This paper focuses on the challenges of choosing emotional } \\
\text { corpora and identification of different hand crafted } \\
\text { features for classification model. }\end{array}$ \\
\hline $\begin{array}{l}\text { Swain et al. [12] } \\
2018\end{array}$ & $x$ & $\checkmark$ & $\checkmark$ & $x$ & $\checkmark$ & $\begin{array}{l}\text { This paper reviews the literature on various databases, } \\
\text { handcrafted features, and classifiers for the SER system. }\end{array}$ \\
\hline $\begin{array}{l}\text { Akccay et al. }[13] \\
2020\end{array}$ & $x$ & $\checkmark$ & $\checkmark$ & $x$ & $\checkmark$ & $\begin{array}{l}\text { This paper focuses on emotional corpora, preprocessing } \\
\text { techniques for handcrafted features, supporting } \\
\text { modalities and emotion classifiers. }\end{array}$ \\
\hline Our paper & $\checkmark$ & $\checkmark$ & $\checkmark$ & $\checkmark$ & $\checkmark$ & $\begin{array}{l}\text { Our paper covers deep different representation learning } \\
\text { techniques from emotional speech in comparison } \\
\text { traditional methods and handcrafted features, covers } \\
\text { poplar emotional corpora, DL models, discusses different } \\
\text { challenges, highlights future directions. }\end{array}$ \\
\hline
\end{tabular}

ing has since evolved significantly with generative models like variational autoencoders (VAEs) [15], and generative adversarial networks (GANs) [16] demonstrating superior performance in representation learning compared to autoencoders and other classical methods [17]. Furthermore, we find surveys that focus on different emotional datasets and handcrafted features and classification networks do not focus on deep representation learning for SER.

We consider multiple databases to find the relevant literature, including IEEE Xplore, Springer, Elsevier, and Google Scholar. We searched articles using related keywords: "representation learning", "feature learning", and "feature extraction". Many studies were also found in the bibliographies of reviewed papers and were included. In general, studies in this review use DL for representation or feature learning/extraction on publicly available datasets.

The major contribution of our paper is that we cover a comprehensive survey that bridges the gaps in the existing literature. More specifically, we focus on (1) the importance of deep representation learning for SER; (2) the popular DL models and their representation learning abilities; and (3) the various representation learning techniques used for SER in the literature. We further highlight the challenges of deep representation learning in SER and conclude this paper by discussing the findings of our review and by identifying future research directions.

The remainder of the paper is organised as follows. We provide a discussion on the relevant background and concepts in Section 2. The use of deep representation learning for SER is discussed in Section 3. The challenges of deep representation learning for SER are discussed in Section 4. Discussions and future directions follows in Section 5. Finally, the paper is concluded in Section 6.

\section{BACKGROUND AND CONCEPTS}

Representation learning has become a rich research discipline in the ML community. In SER, representation learning can use raw speech as well as speech features to learn emotionally discriminating representations for emotion classification or prediction (as highlighted in Figure 1). This section briefly discusses various important concepts related to representation learning in SER.

\subsection{Representation Learning Vs Feature Engineering}

The manual design of a conversion of the speech signal into meaningful information and a reasonably limited number of attributes using domain knowledge is called (speech) feature engineering. In SER, feature engineering and designing ML models for classification or prediction are often considered separate problems. Most of the actual SER research has focused on feature engineering or the design of pre-processing data transformation pipelines to craft emotional representations that support ML algorithms. Although feature engineering techniques can help improve the SER performance, the downside is that these techniques are labour-intensive and time-consuming. For decades, Mel frequency cepstral coefficients (MFCCs) [18] has been used as the principal set of features for SER and other speech analysis tasks. The four steps involved in the extraction of MFCCs are: (1) computation of the Fourier transform, (2) projection of the powers of the spectrum onto the Mel scale, (3) taking the logarithm of the Mel frequencies, and (4) applying discrete cosine transformation (DCT) or other suited transformations for compressed representations. It is found that the last step loses information and destroys spatial relations; therefore, it is usually omitted, which results in the LogMel spectrum, a popular feature used by the speech community. It is also the most popular feature to train DL networks in the speech domain. Minimalist feature sets like GeMAPs and eGeMAPs [19] are also widely used (e. g., [20], [21]) as benchmarks. They are designed/engineered to (a) index affective physiological changes in voice production, and (b) achieve automatic extractability [19].

On the other hand, representation learning is the technique of learning representations, usually through the automatic transformation of the input data. It comes under the header of DL or feature learning. The key goal of representation learning is yielding abstract and useful representations for ML tasks such as classification and prediction. We compare feature engineering with representation learning in Table 2. The comparisons show that representation learning 
is a less time consuming automatic process and requires minimal human domain knowledge to produce better results than the hand-engineered features. Also, unlike feature engineering, representation learning does not require extra efforts to design features for a new task and have more generalisation ability.

TABLE 2: Comparing feature engineering and representation learning

\begin{tabular}{|c|c|c|c|}
\hline & Automated & Human Independence & Generalisability \\
\hline Feature Engineering & $x$ & $x$ & $x$ \\
\hline Representation Learning & $\checkmark$ & $\checkmark$ & $\checkmark$ \\
\hline
\end{tabular}

\subsection{Traditional vs Deep Representations Learning Techniques}

In the field of representation learning, the algorithms are generally categorised into two classes: shallow and deep [22]. Shallow learning algorithms are also considered as traditional methods. They aim to learn transformations of data by extracting useful information. One of the oldest shallow learning algorithms is PCA [14], which has been studied extensively over the last century. Similar to PCA, linear discriminant analysis (LDA) [23] is another shallow learning algorithm. Unlike PCA, LDA is a supervised method that requires class labels to maximise class separability. Other linear feature learning methods include canonical correlation analysis (CCA) [24], multi-dimensional scaling (MDS) [25], and independent component analysis (ICA) [26].

Many methods for nonlinear feature reduction are also proposed to discover the non-linear hidden structure from the high dimensional data [27] including locally linear embedding (LLE) [28], non-negative sparse coding [29], isometric feature mapping (Isomap) [30], t-distributed stochastic neighbour embedding (t-SNE) [31], and neural networks (NNs) [32]. The kernel PCA (KPCA) [33], and generalised discriminant analysis (GDA) [34] are non-linear versions of PCA and LDA, respectively.

The shortcoming of shallow representation learning is that such representations contain only a small number of non-linear operations and are unable to accurately model complex, high-dimensional, and noisy real-world data (such as emotional speech) [35]. The shallow feature learning algorithms have, however, dominated representation learning until the successful training of deep models for representation learning of data reported by Hinton and Salakhutdinov in 2006 [36]. This work was quickly uptaken by other researchers [37], [38], which led to a large number of deep models suitable for deep representation learning. In DL models (e.g., feed-forward neural networks), all hidden layers except the last layer (i. e., softmax classifier) learn representations, which often leads to much better performance compared to the hand-designed representations [39].

Studies show that deep architectures can learn more complex relationships that greatly help improve performance [22]. The non-linearity in deep models help to learn more robust representations when multiple layers/modules are stacked atop one another. Such robust representation in lower dimensions can be easily transmitted to the communication network for a wide range of real-time applications and services [40].

\subsection{Deep Learning Models for Representation Learning}

This section covers DL models, including feed-forward neural networks (FNNs), autoencoders, and generative models, which have been widely used for emotional representation learning in SER research. We highlight the characteristics of these models in terms of their emotional representation learning abilities in Table 3.

TABLE 3: Characteristics of DL model for representation learning. Here DNN represents fully connected deep networks.

\begin{tabular}{|l|l|}
\hline Model & Characteristics \\
\hline DNNs & $\begin{array}{l}\text { Good for learning a hierarchy of representations. They can learn } \\
\text { invariant and discriminative representations. Features learnt } \\
\text { by DNNs are more generalised compared to traditional methods. }\end{array}$ \\
\hline CNNs & $\begin{array}{l}\text { Good for learning both low-level as well as high-level } \\
\text { representation from emotional speech. }\end{array}$ \\
\hline RNNs & $\begin{array}{l}\text { Good for sequential modelling. They can learn temporal } \\
\text { structures from speech suitable for emotion classification. }\end{array}$ \\
\hline AEs & $\begin{array}{l}\text { Powerful unsupervised representation learning models that } \\
\text { encode the emotional speech data in sparse and compressed } \\
\text { representations. }\end{array}$ \\
\hline VAEs & $\begin{array}{l}\text { Stochastic variational inference and learning model. Popular } \\
\text { in learning disentangled emotional representations from speech. }\end{array}$ \\
\hline GANs & $\begin{array}{l}\text { A Game-theoretical framework that is useful for data } \\
\text { generation and is robust to overfitting. They can learn } \\
\text { disentangled representations that are very suitable for SER. }\end{array}$ \\
\hline
\end{tabular}

\subsubsection{Deep Neural Networks (DNNs)}

Historically, the idea of fully connected $\mathrm{DNNs}^{1}$ is an extension of ideas emerging from artificial neural networks (ANNs) [41]. Multilayer perceptrons (MLPs) [42] with multiple hidden layers are indeed a good example of deep architectures. DNNs consist of multiple layers, including an input layer, hidden layers, and an output layer, of processing units called "neurons". These neurons in each layer are densely connected with the neurons of the adjacent layers. Each layer of a DNN performs representation learning based on the input provided to it. A well trained DNN learns a hierarchy of distributed representations [43]. Increasing the depth of DNNs enables the learning of a deep hierarchy of representations at different levels of abstraction. DNN with highway connectivity [44] is a good example of such deep architecture. Higher levels of abstract representations generally offer invariance to local changes of the input [9] and are helpful in designing SER systems.

Convolutional neural networks (CNNs) [45] are a specialised kind of deep architecture for processing data having a grid-like topology. Examples include image data that have $2 \mathrm{D}$ grid pixels and time-series data (i.e., 1D grid). CNNs introduce convolutional, and pooling layers into the structure of DNNs, which take into account the spatial representations of the data and make the network more efficient by introducing sparse interactions, parameter sharing, and equivariant representations [46]. There are many variants of CNNs; however, ResNet [47], and DenseNet [48] are especially popular in SER due to their complex emotional representation learning ability.

In contrast to fully connected DNNs, the training process of CNNs is more straightforward due to fewer parameters [49]. CNNs are powerful at extracting low-level representations at the initial layers, and high-level features (textures and semantics) in the higher layers [50]. The convolution layer in CNNs acts as a data-driven filterbank that is able to

1. We use DNNs for fully connected deep neural networks. 
capture emotional representations from speech, which are more generalised, discriminative, and contextual [50], [51].

Recurrent neural networks (RNNs) [52], [53] introduce recurrent connections within layers to enable parameters sharing across time. They create a memory in the network by using the information from all previous inputs. This makes RNNs have stronger representational memory compared to hidden Markov models (HMMs), whose discrete hidden states bound their memory [54]. Simple RNNs usually fail to model the long-term temporal contingencies due to the vanishing gradient problem. Multiple specialised RNN architectures, including long short-term memory (LSTM) [55] and gated recurrent units (GRUs) [54] address this problem using a gating mechanism to add and forget the information selectively. Bidirectional RNNs [56] also model both past and future contexts by passing the input sequence through two separate recurrent hidden layers. RNNs introduce recurrent connections to allow parameters to be shared across time, making them powerful in learning temporal dynamics from sequential data, e.g., speech. In SER, temporal dynamics modelling using RNNs-Connectionist Temporal Classification (CTC) [57] based models shows improved results.

\subsubsection{Autoencoders (AEs)}

The idea of an autoencoding network [58] is to learn a mapping from high-dimensional data to a lower-dimensional feature space such that the input observations can be approximately reconstructed from the lower-dimensional representation. A function $f_{\theta}$ called the encoder maps the input vector $x$ into feature/representation vector $h=f_{\theta}(x)$. The decoder network is responsible to map a feature vector $h$ to reconstruct the input vector $\hat{x}=g_{\theta}(h)$. The decoder network parameterises the decoder function $g_{\theta}$. Overall, the parameters are optimised by minimising the following cost function:

$$
\mathcal{L}\left(x, g_{\theta}\left(f_{\theta}(x)\right)\right)=\|x-\hat{x}\|_{2}^{2} .
$$

The set of parameters $\theta$ of the encoder and decoder networks are simultaneously learned by incurring a minimal reconstruction error. To capture useful representations $h$, the cost function of Equation 1 is usually optimised with an additional constraint to prevent the AE from learning the useless identity function having zero reconstruction error. One way of learning useful feature representations $h$ is to regularise the autoencoder by imposing constraints to have a low dimensional feature size. In this way, the AE is forced to learn the salient representations of data from a high dimensional space to a low dimensional feature space. Below we discuss some other autoencoding networks.

Sparse autoencoders (AEs) can discover a useful feature representation with the size larger than the input vector $x$ [59]. This is done using the sparsity regularisation [38]. Sparseness plays a key role in learning a more meaningful representation of input data [60]. It has been found that sparse AEs are simple to train and can learn better representation compared to denoising autoencoders (DAE) and RBMs [61]. In particular, sparse encoders can learn useful information and attributes from emotional speech, which can facilitate better classification performance [62].

Denoising autoencoders (DAEs) are considered as a stochastic version of the basic AE. They are trained to reconstruct a clean input from its corrupted version [63]. The objective function of a DAE is given by:

$$
\mathcal{L}\left(x, g_{\theta}\left(f_{\theta}(\tilde{x})\right)\right),
$$

where $\tilde{x}$ is the corrupted version of $x$, which is done via stochastic mapping $\tilde{x} \sim q_{D}(\tilde{x} \mid x)$. During training, DAEs minimise the same reconstruction loss between a clean $x$ and its reconstruction from $h$. The difference is that $h$ is learnt by applying a deterministic mapping $f_{\theta}$ to a corrupted input $\tilde{x}$. It thus learns higher level feature representations that are robust to input corruption. Therefore, DAEs are suitable for learning emotional representations from noisy speech [64].

\subsubsection{Deep Generative Models}

Deep belief network (DBN) [65] is a powerful probabilistic generative model that consists of multiple layers of stochastic latent variables, where each layer is a restricted Boltzmann machine (RBM) [66]. Boltzmann machine (BM) is a bipartite graph in which visible units are connected to hidden units using undirected connections with weights. A $\mathrm{BM}$ is restricted in the sense that there are no hidden-hidden and visible connections. During the training phase, an RBM uses Markov chain Monte Carlo (MCMC)-based algorithms [65] to maximise the log-likelihood of the training data. RBMs are very effective at approximating any distribution. However, training RBMs based on MCMC computes the gradient of the log-likelihood, which poses a significant learning problem [67]. In recent years, generative models like GANs and VAEs have been proposed that can be trained via direct back-propagation and avoid the difficulties of MCMC-based training. We discuss GANs and VAEs in more detail next.

Generative adversarial networks (GANs) [16] use adversarial training to directly shape the output distribution of the network via back-propagation. They include two neural networks-a generator, $G$, and a discriminator, $D$, which play a min-max adversarial game defined by the following optimisation problem:

$$
\min _{G} \max _{D} \mathrm{E}_{x}[\log (D(x))]+\mathrm{E}_{z}[\log (1-D(G(z)))] .
$$

The generator, $G$, maps the latent vectors, $z$, drawn from some known prior, $p_{z}$ (e.g., Gaussian), to fake data points, $G(z)$. The discriminator, $D$, is tasked to differentiate between generated samples (fake), $G(z)$, and real data samples, $x \in p_{\text {data }}$. Overall, GAN is trained to achieve a generator network that maximally confuses the discriminator into believing that the samples it generates come from the data distribution.

Benefiting from the flexibility of GAN's framework, adversarial training methodology has been successfully leveraged to many traditional tasks, including unsupervised representation learning. For example, DCGAN [17] uses the intermediate features from the discriminator as the representations of the input images. On the other hand, the input of the generator, i.e., noises, can be viewed as the representations of the output images.

Variational autoencoders (VAEs) are probabilistic models that use a stochastic encoder for modelling the posterior distribution $q(z \mid x)$, and a generative network (decoder) that models the conditional Log-likelihood $\log p(x \mid z)$. Both of 
these networks are jointly trained to maximise the following variational lower bound on the data loglikelihood:

$$
\log p(x)>\mathbb{E}_{q(z \mid x)} \log p(x \mid z)-\mathrm{KL}(q(z \mid x)|| p(z)) .
$$

The first term is the standard reconstruction term of an AE, and the second term is the KL divergence between the prior $p(z)$ and the posterior distribution $q(z \mid x)$. The second term acts as a regularisation term, and without it, the model is simply a standard autoencoder. In contrast to standard AEs, VAEs learn the probability distribution parameters from the input in a latent space by making the latent distribution close to a 'prior' distribution. Due to these characteristics, VAEs are becoming very popular in learning emotional representation from speech [68]. Recently, various variants of VAEs are proposed in the literature, which include $\beta$-VAE [69], InfoVAE [70], FactorVAE [71], and many more [72]. All these VAEs are very powerful in learning disentangled, and hierarchical representations and are also popular in clustering multi-category structures of data [72].

\subsection{Emotional Corpus}

Emotional databases can be divided into three types: simulated, elicited (induced), and natural. Simulated emotional speech databases consist of recordings collected from experienced and trained actors or artists. Induced emotional speech databases are collected by generating an emotional situation artificially by involving the speaker in the emotional dialogue or conversational setting. The speaker's reactions towards the emotional situation are potentially recorded without their knowledge after ethical approval. Natural emotional databases are produced by recording emotions from real-world applications such as call centres [73], or patient and doctors conversations, among others. Natural emotional databases may not contain all emotions and also have copyright and privacy issues.

Different emotional datasets are available; however, in this work, we only present the details of the most popular ones in Table 4, which are being utilised for emotional representation learning. In [74], [75], the authors provide further details on speech emotional databases. These emotional datasets are annotated using either categorical [76] or dimensional [77] emotion models. A categorical emotion model considers emotions as discrete classes, whereas a dimensional emotion model defines emotions as two or more dimensional space characterised mostly by arousal and valence and next frequently dominance.

\subsection{Evaluation Metrics}

In SER, the effectiveness of a deep emotional representation is evaluated by performing classification or regression using these representations as input. For classification, SER systems use a classification score or accuracy as a metric. However, as data is often imbalanced across the classes, in naturalistic emotion corpora, the accuracy is usually used as so-called unweighted accuracy (UA) or unweighted average recall (UAR), representing the average recall across classes, unweighted by the number of instances per classes. This has been introduced by the first challenge in the fieldthe Interspeech 2009 Emotion Challenge [86] and has since been used by other challenges across the field. SER systems that use deep representation for emotional attributes such as arousal and valence or dominance prediction commonly optimise regression-based models using the mean squared error (MSE) and concordance correlation coefficient (CCC) as objective functions [87].

\section{Deep Representation Learning in SER}

In this section, we review the existing literature on deep representation learning techniques for SER. The readers are referred to Table 5 for a summary of the reviewed studies in this paper. We present studies while describing their use of corpus, input features, models, and performance in Table 5. Studies are clustered into five major groups depending on the DL techniques employed for representation learning:

\subsection{Supervised Representation Learning}

In supervised representation learning, features are learnt from data samples using their labels. In SER, supervised representation learning methods are widely used to improve performance. In [6], the authors use a DBN for emotional representation learning from speech and achieve $7 \%$ higher classification accuracy $(86.5 \%)$ on the BUAA emotional corpus compared to the classical hand-engineered features. To improve the SER performance, Cairong et al. [7] fuse the classical features with emotional representation learnt by a DBN. They show that the fusion of deep emotional representations learnt by a DBN with classical features can improve SER by $8.8 \%$. A similar fusion of a DBN representation with hand-engineered features was performed in [88] to improve SER performance in noisy conditions. Experiments were performed on the EMODB dataset, and the results show that the proposed approach improves the performance by $5.48 \%$. In [89], the authors perform experiments on multiple datasets and show that DBNs can learn more powerful and effective discriminative long-range features that help improve SER performance. Similar to DBNs, researchers also explored DNNs with multiple fully connected hidden layers for emotional representation learning.

Deep neural networks (DNNs) are popular in learning high-level discriminative emotional representations. Hen et al. [90] use DNNs for high-level emotional representation learning from raw speech. They construct an utterance-level representation from a segment level probability distribution produced by a DNN and use extreme learning machines (ELMs) to perform emotion classification on these utterance level representations. They evaluate the proposed framework on the IEMOCAP data and show that the proposed approach effectively captures emotional representation and leads to $20 \%$ classification improvement. In [91], the authors attempt to learn a discriminative emotional representation in compressed size to facilitate fast classification. The results show that DNNs can capture a hidden emotional representation that leads to significant improvement in SER performance. Various other studies [92]-[94] also explore DNNs for emotional representation from speech; however, the improved ability of RNNs for better modelling of longrange emotional context shift the research towards using RNNs in state-of-the-art SER systems.

Recurrent neural networks (RNNs) with gated architectures are specialised to model a long-range of contexts. Emotions in human speech are contextually embedded; therefore, the context capturing abilities of RNNs such as 
TABLE 4: Review of different SER databases.

\begin{tabular}{|c|c|c|c|c|c|c|c|}
\hline Corpus Name & Language & Speakers & Mode & Type & Emotions & $\begin{array}{l}\text { Duration } \\
\text { (approx) }\end{array}$ & Public Access \\
\hline EMODB [78]) & German & $\begin{array}{l}10 \text { speakers ( } 5 \text { males, } 5 \\
\text { females) }\end{array}$ & audio & stimulated & $\begin{array}{l}\text { anger, boredom, disgust, } \\
\text { fear, happiness, sadness, } \\
\text { neutral }\end{array}$ & $<1$ hour & yes \\
\hline MSP-IMPROV [79] & English & $\begin{array}{l}12 \text { actors ( } 6 \text { males and } \\
6 \text { females) }\end{array}$ & audio, video & stimulated & $\begin{array}{l}\text { anger, happiness, } \\
\text { sadness, neutral }\end{array}$ & 18 hour & yes \\
\hline MSP-Podcast [80] & English & $\begin{array}{l}60 \text { speakers ( } 30 \text { females, } \\
30 \text { males) }\end{array}$ & audio & naturalistic & arousal, valence, dominance & 27 hours & yes \\
\hline SEMAINE [81] & English & 150 participants & audio, video & induced & $\begin{array}{l}5 \text { affective dimensions } \\
\text { (i. e., valence, activation, } \\
\text { power, anticipation/ } \\
\text { expectation, } \\
\text { intensity) }\end{array}$ & 6.2 hours & yes \\
\hline IEMOCAP [82] & English & 5 females, 5 males & audio, video & stimulated & $\begin{array}{l}\text { neutral, happiness, sadness, } \\
\text { anger, surprise, fear, } \\
\text { disgust, frustration, excited } \\
\text { and other) }\end{array}$ & 12 hours & yes \\
\hline EMOVO [83] & Italian & $\begin{array}{l}6 \text { speakers ( } 3 \text { males, } \\
3 \text { females) }\end{array}$ & audio & stimulated & $\begin{array}{l}\text { disgust, happiness, fear, } \\
\text { anger, surprise, sadness, } \\
\text { neutral }\end{array}$ & $<2$ hours & yes \\
\hline RECOLA [84] & French & $\begin{array}{l}46 \text { speakers ( } 19 \text { males, } \\
27 \text { females) }\end{array}$ & audio, video & natural & $\begin{array}{l}\text { five social behaviours } \\
\text { (dominance, agreement, } \\
\text { performance, engagement, } \\
\text { rapport); arousal and } \\
\text { valence }\end{array}$ & 3.5 hours & Yes \\
\hline CMU-MOSEI [85] & English & single speaker & multimodal & natural & $\begin{array}{l}\text { anger, anxious, disgust, } \\
\text { happiness, neutral, sadness, } \\
\text { surprise and fear }\end{array}$ & 65 hours & Yes \\
\hline
\end{tabular}

LSTM and GRU were explored in SER research by various studies. Lee et al. [8] use RNNs to learn high-level temporal dynamics of emotional representation from speech. They adopt bidirectional long short-term memory (BLSTM) network and achieve $12 \%$ improved results compared to the DNN-ELM [90]. In [124], the authors evaluate different BLSTM-RNN architectures for emotional representation learning from speech. They use the IEMOCAP corpus for evaluation and found that RNNs can learn both emotionally relevant short-term frame-level acoustic representation and compact utterance-level emotional representation of framelevel features. They report that BLSTMs outperform DNNs and SVMs trained on hand-engineered features. Ghosh et al. [98] evaluate the representation learning from spectrogram and glottal flow signals using DAE-BLSTM models. They report that their proposed framework can generate highly discriminative representations that produce comparable emotion classification results to state-of-the-art approaches.

Convolutional neural networks (CNNs) are also popular for emotional representation learning in SER [5], [125][128]. They can learn more generalised features from speech compared to DNNs, and other feature-based approaches [50]. In [96], the authors explore CNN for speech emotion detection. They found that CNN filters capture emotions related to the fundamental frequency, which helps create discriminative features for SER. Feature representations learnt by deep CNNs show robustness against noisy situations [129], [130]. Therefore, studies in SER also use deep CNN architectures such as ResNet and DenseNet for SER in noisy environment [131], [132]. The research on emotional representation learning was further advanced by the use of combined CNN and RNN architectures. Various studies used CNN-LSTM [50], [87], [133], [134], where they used CNNs for feature extraction and LSTM (or GRU) networks for modelling long term dependencies. Based on the results, these studies showed that CNN-RNNs is a better choice in contrast to using CNN or LSTM individually. In order to learn the spatial relationships in spectrograms, the authors in [135] used capsule networks (CapsNets). They designed a sequential capsule structure to obtain utterancelevel emotional representations and evaluated the proposed model on the IEMOCAP dataset. They compared the results with baseline CNN-LSTM and showed that the proposed CapsNets are able to produce improved results in SER compared to the CNN-LSTM.

In supervised representation learning, attention-based networks have recently become very popular. Attention layers in DL models help the networks to focus on important emotional representations in the input speech. Researchers have attempted various attention mechanisms including self-attention [136], local attention [124], multi-hop attention [137], and many other variants [113], [138]. In these studies, the authors show that attention mechanisms enable networks to focus on affect-salient components and extract emotional representations from speech sentences, which help to improve the performance of the system.

Despite the promising results, the success of supervised training is limited by the requisite of labels. It is important to note that creating and labelling these datasets is very expensive in terms of time and resources. To tackle these issues, unsupervised learning has been used to learn representations from unlabelled data. We discuss unsupervised representation learning of speech emotion in the next subsection.

\subsection{Unsupervised Representation Learning}

Unsupervised representation learning facilitates the analysis of input data without corresponding labels and aims to learn the underlying inherent structure or distribution of the data. Real-life data (such as speech, image, or text) have extremely rich structures. Algorithms trained in an unsupervised way aim to learn the underlying structure of the data rather than learning any particular tasks, e. g., classification, prediction etc. In speech analysis, unsupervised representation learning can exploit the unlimited amount of unlabelled corpora 
TABLE 5: Summary of deep representation learning techniques used for SER in different studies. C: classification, P: prediction, ARR: Average Recognition Rate, AAC: Average Accuracy, IS09-IS13: Interspeech 2009-2013 paralinguistics challenge feature set, LLDs: Low-Level Descriptors, act: activation, val: valence, dom: dominance, aro: arousal.

\begin{tabular}{|c|c|c|c|c|c|}
\hline Paper (Year) & Technique & Corpus & Input & Model (C/P) & Performance \\
\hline Cairong et al. [7] (2014) & \multirow{7}{*}{$\begin{array}{l}\text { Supervised } \\
\text { Representation } \\
\text { Learning }\end{array}$} & ABC corpus [95] & Spectrogram & $\mathrm{DBN}(\mathrm{C})$ & $52.2 \%(\mathrm{ARR})$ \\
\hline Kim et al. [94] (2019) & & IEMOCAP & $\begin{array}{l}\text { acoustic +lexical } \\
\text { features }\end{array}$ & $\mathrm{DNN}(\mathrm{C})$ & $61.4 \%$ (UAR) \\
\hline Fayek et al. [93] (2017) & & IEMOCAP & Mel scale features & DNN (C) & $58.78 \%$ (UAR) \\
\hline Bertero et al.[96] (2017) & & TED talks [97] & Raw speech & CNN (C) & $66.1 \%(\mathrm{AAC})$ \\
\hline Aldeneh et al. [51] (2017) & & $\begin{array}{l}\text { IEMOCAP } \\
\text { MSP-IMPROV }\end{array}$ & Mel filterbanks & CNN (C) & $\begin{array}{l}61.8 \% \text { (UAR) } \\
52.6 \% \text { (UAR) }\end{array}$ \\
\hline Lee et al. [8] (2015) & & IEMOCAP & Acoustic features & BLSTM (C) & $63.89 \%(\mathrm{UA})$ \\
\hline Latif et al. [50] (2019) & & $\begin{array}{l}\text { IEMOCAP } \\
\text { MSP-IMPROV }\end{array}$ & Raw Speech & CNN-LSTM & $\begin{array}{l}60.23 \% \text { (UAR) } \\
52.43 \% \text { (UAR) }\end{array}$ \\
\hline Ghosh et al. [98] (2016) & & IEMOCAP & Spectrogram & DAE-BLSTM (C) & $51.86 \%$ (UA) \\
\hline Xia et al. [99] (2016) & & IEMOCAP & IS10 [100] & DAE-SVM (C) & 63.1\% (UAR) \\
\hline $\begin{array}{l}\text { Paraskevopoulos et al. [101] } \\
\text { (2019) }\end{array}$ & & IEMOCAP & IS10 & AE-SVM (C) & $57.8 \%$ (UA) \\
\hline Latif et al. [68] (2018) & & IEMOCAP & LogMel features & VAE-LSTM (C) & $55.42 \%$ (UA) \\
\hline Eskimez et al. [102] (2018) & & IEMOCAP & $\begin{array}{l}\text { MFCCs +LLDs } \\
\text { and derivatives }\end{array}$ & AAE-DNN (C) & $48.18 \%$ (UAR) \\
\hline Latif et al. [103] (2020) & & IEMOCAP & IS10 & GAN-DNN (C) & $60.51 \%$ (UAR) \\
\hline $\begin{array}{l}\text { Parthasarathy et al. [104] } \\
\text { (2018) }\end{array}$ & & MSP-Podcast & IS13 [105] & $\begin{array}{l}\text { Ladder } \\
\text { networks }(\mathrm{P})\end{array}$ & $\begin{array}{l}\text { aro }(0.803 \mathrm{CCC}) \\
\text { val }(0.458 \mathrm{CCC}) \\
\operatorname{dom}(0.746)\end{array}$ \\
\hline $\begin{array}{l}\text { Parthasarathy et al. [106] } \\
(2020)\end{array}$ & $\begin{array}{l}\text { Semi-supervised } \\
\text { Representation } \\
\text { Learning }\end{array}$ & MSP-Podcast & IS13 & $\begin{array}{l}\text { Ladder } \\
\text { networks (P) }\end{array}$ & $\begin{array}{l}\text { aro }(0.770 \text { CCC }) \\
\text { val }(0.301 \text { CCC }) \\
\text { dom }(0.700)\end{array}$ \\
\hline $\begin{array}{l}\text { Tao et al. [107] } \\
\text { (2019) }\end{array}$ & & IEMOCAP & IS09 [108] & $\begin{array}{l}\text { Ladder } \\
\text { networks (C) }\end{array}$ & $59.7 \%$ (UAR) \\
\hline Latif et al. [109] (2020) & & $\begin{array}{l}\text { IEMOCAP } \\
\text { MSP-IMPROV }\end{array}$ & Spectrogram & AAE-CNN (C) & $\begin{array}{l}66.7 \% \text { (UA) } \\
60.3 \% \text { (UA) }\end{array}$ \\
\hline Chang et al.[110] (2017) & & IEMOCAP & Spectrogram & GAN-DNN (C) & $48.88 \%$ (UA) \\
\hline $\begin{array}{l}\text { Xia et al. [111] } \\
(2017)\end{array}$ & & $\begin{array}{l}\text { IEMOCAP } \\
\text { SEMAINE }\end{array}$ & IS10 & $\mathrm{DBN}(\mathrm{C})$ & $\begin{array}{l}60.5 \% \text { (UA) } \\
35.9 \% \text { (UA) }\end{array}$ \\
\hline $\begin{array}{l}\text { Lotfian et al. [112] } \\
\text { (2018) }\end{array}$ & $\begin{array}{l}\text { Multi-task } \\
\text { Representation }\end{array}$ & MSP-Podcast & eGeMAPS & $\mathrm{DNN}(\mathrm{C})$ & $66.8 \%$ (UA) \\
\hline $\begin{array}{l}\text { Nediyanchath et al. [113] } \\
(2020)\end{array}$ & Learning & IEMOCAP & LogMel & $\mathrm{DNN}(\mathrm{C})$ & $70.1 \%$ (UA) \\
\hline Tao et al.[114] (2018) & & IEMOCAP & IS10 & LSTM (C) & $55.3 \%(\mathrm{WA})$ \\
\hline $\begin{array}{l}\text { Parthasarathy et al. [115] } \\
\text { (2017) }\end{array}$ & & GeWEC [116] & IS09 & Universum AE (C) & $63.3 \%$ (UAR) \\
\hline $\begin{array}{l}\text { Abdelwahab et al. [117] } \\
\text { (2018) }\end{array}$ & $\begin{array}{l}\text { Domain } \\
\text { Adaptive } \\
\text { Representation }\end{array}$ & $\begin{array}{l}\text { IEMOCAP } \\
\text { MSP-IMPROV }\end{array}$ & IS13 & DANN (P) & $\begin{array}{l}\text { aro }(.489 \text { CCC }) \\
\text { val }(.215 \text { CCC }) \\
\text { dom }(.401 \text { CCC })\end{array}$ \\
\hline $\begin{array}{l}\text { Paraskevopoulos et al. [101] } \\
(2019)\end{array}$ & & $\begin{array}{l}\text { IEMOCAP } \\
\text { MSP-IMPROV }\end{array}$ & IS09 & $\mathrm{DNN}(\mathrm{C})$ & $\begin{array}{l}56.2 \% \text { (UAR) } \\
44.1 \% \text { (UAR) }\end{array}$ \\
\hline $\begin{array}{l}\text { Shukla et al. [118] } \\
(2021)\end{array}$ & Self Supervised & IEMOCAP & Spectrogram & CNN-LSTM(C) & $0.615 \%(\mathrm{~F} 1)$ \\
\hline $\begin{array}{l}\text { Siriwardhana et al. [119] } \\
(2020)\end{array}$ & $\begin{array}{l}\text { Representation } \\
\text { Learning }\end{array}$ & IEMOCAP & Wav2Vec [120] & Transformer (C) & 74.7 (AAC) \\
\hline $\begin{array}{l}\text { Lakomkin et al. [121] } \\
\text { (2021) }\end{array}$ & DRL for & IEMOCAP & MFCC & GRU (C) & $84.9 \%$ AAC \\
\hline $\begin{array}{l}\text { Chen et al. [122] } \\
(2019)\end{array}$ & $\begin{array}{l}\text { Representation } \\
\text { Learning }\end{array}$ & CMU-MOSEI & $\begin{array}{l}\text { MFCCs } \\
\text { HOG [123] }\end{array}$ & Multimodal-LSTM (C) & $76.5 \%(\mathrm{AAC})$ \\
\hline
\end{tabular}

to learn good intermediate feature representations, which can then be used to improve the performance of supervised SER, where availability of labelled data is limited [139].

Autoencoders (AEs) are mostly utilised for unsupervised emotional representation learning from speech in SER. AEs can learn high-level semantic contents that are invariant to confounding low-level details (pitch contour or background noise) in speech [140]. In [141], the authors explored Denoising Autoencoders (DAEs) for emotional representation learning on IEMOCAP data. They empirically showed that a representation captured by the bottleneck layer of AEs are highly discriminative in separating the emotions and help to achieve comparable results to that of using hand-engineered features (such as voice quality features and MFCCs). In [98], Ghosh et al. use stacked DAEs for learning frame-level emotional representations from the spectrogram of speech and glottal flow signals. They evaluated the proposed framework on IEMOCAP data and found that a stacked DAE can learn highly discriminative features that help to achieve state-of-the-art results (54.56\% UA). Huang et al. [142] evaluated different unsupervised representation learning algorithms including K-means clustering, the sparse AE, and sparse RBMs for SER. They explored the effect of the content window size and the number of hidden nodes on the performance. They found that a larger content window and more hidden units produce better results. To extract robust features, Xia et al. [64] used DAEs for SER. The authors empirically showed that DAEs can extract more robust feature representations and significantly outperformed using the static features for SER. Another work [99] utilised a modified DAE to model gender information to learn more robust emotional representations. The authors evaluated the proposed model on IEMOCAP data achieving improved results compared to the DAE used in [64] and hand-engineered features. Models like RBMs and DBNs can also learn high-level feature representations [143] and auditory-like sub-band filters [144] from speech, which can help improve the performance compared to 
hand-engineered features when used in SER for unsupervised representation learning [6], [145]. In [146], the authors utilise unsupervised learnt representations from unlabelled data to improve SER. They integrate an AE with a CNNbased emotion classifier to improve SER performance for within-corpus and cross-corpus settings. Generative models have further advanced the performance of unsupervised representation learning in SER.

Generative models including VAEs, GANs, and adversarial autoencoders (AAE), are becoming very popular in emotional representation learning due to their exceptional performance in learning representation and generating new data samples. In [68], the authors explore VAE architectures for latent representations of speech emotion. They perform extensive experiments on the IEMOCAP dataset and show that VAEs can learn discriminative emotional attributes suitable for improving classification than standard AEs. Eskimez et al. [102] evaluate various unsupervised autoencoding networks including DAE, VAE, AAEs, and adversarial variational Bayes (AVB) for emotional representation learning. They find that these unsupervised methods can capture the intrinsic structures of speech emotion that help to achieve improved results compared to SVMs and CNNs. Latif et al. [103] use GANs for representation learning as well as generating synthetic representations. They modify the GAN and use the mixup [147] technique to augment a GAN in SER. They achieve $61.05 \%$ and $46.60 \%$ accuracy on within-corpus and cross-corpus settings, respectively. These studies show that generative models can learn better emotional representations and generate synthetic data samples that can be used to train the classifier along with real samples. This can lead to improvements in SER performance even in low resource conditions.

Despite all these successes, the performance of unsupervised representation learning techniques is not as good as that of the supervised methods [109]. Semi-supervised representation learning techniques alleviate this problem by simultaneously utilising both labelled and unlabelled data. We discuss the semi-supervised representation learning technique in the next subsection.

\subsection{Semi-supervised Representation Learning}

The success in DL has predominately been possible due to key factors like advanced algorithms, processing hardware, open sharing of codes and papers, and most importantly, the availability of large-scale labelled datasets (e.g., ImageNet) pre-trained networks. However, a large labelled database or pre-trained network for every problem like SER is not always available [20], [89], [148]. It is very difficult, expensive, and time-consuming to annotate speech emotional data as it requires manual expert human efforts [109]. Semisupervised representation learning attempts to solve this problem by utilising the feature representations from large unlabelled data, jointly with the labelled data, to build better classifiers. It reduces human efforts and provides higher accuracy in contrast to unsupervised representation learning; therefore, semi-supervised models are of great interest both in theory and practice [149]. Huang et al. [150] use a $\mathrm{CNN}$ in a semi-supervised way for capturing affect-salient representations. They evaluate their model on four publicly available datasets and find that a semi-supervised CNN learned salience, orthogonal, and discriminative representations for SER. These representations help to achieve superior performance compared to results using well-established hand-engineered features. Deng et al. [151] propose a semisupervised model by combining an AE and a classifier. They consider samples from unlabelled data as an extra garbage class in the classification problem. They evaluate the proposed architecture on five publicly available datasets and show that features learnt by a semi-supervised AE improve SER performance compared to an unsupervised AE. Parthasarathy et al. [152] utilise semi-supervised AAE to disentangle the discrete emotion distribution and show that the proposed learning approach performs better compared to a fully supervised method. In [109], the authors train an AAE by utilising the additional unlabelled emotional data to improve SER performance. They perform evaluations on IEMOCAP and MSP-IMPROV and show that additional data help to learn more generalised representations that perform better than various supervised and unsupervised methods.

Ladder network-based semi-supervised methods are very popular in SER. A ladder network is an unsupervised DAE that is trained along with a supervised classification or regression task. It can learn more generalised representations suitable for SER compared to the standard methods. Parthasarathy et al. [104] use a ladder network with skip connections between encoder and decoder networks for emotional representation learning. They evaluate the proposed model on the MSP-Podcast dataset and find that a semi-supervised ladder network can lean more powerful representations that facilitate better performance in predictions of emotional attributes than conventional DAE. In another study [106], the authors show that a ladder network can generate powerful and generalised representations that help to achieve relative gains in concordance correlation coefficient (CCC) of $3.0 \%$ to $3.5 \%$ for within-corpus, and $16.1 \%$ to $74.1 \%$ for cross-corpus settings using the MSPPodcast, IEMOCAP, and MSP-IMPROV datasets. In [153], the authors utilise a semi-supervised ladder network to generate a robust feature representation by simultaneously minimising the sum of supervised classification and unsupervised cost functions. The features generated by a ladder network are used as an emotional representation for classification with an SVM. They perform evaluations on the IEMOCAP corpus and show that the proposed framework achieves $2.6 \%$ improved performance than a DAE, and $5.3 \%$ higher than the static acoustic features. Tao et al. [107] also utilise a ladder network for emotional representation generation and conducted experiments on the IEMOCAP dataset, and achieve improved classification performance with a small number of labelled samples compared to DAE, VAE, and hand-engineered features.

Generative adversarial networks (GANs) were also explored for semi-supervised representation learning in SER. Chang et al. [110] performed emotional representation learning from speech using a convolutional GAN. They utilise 100 hours of unlabelled data and show that the proposed model derives automatic discriminative representations learning to improve the SER performance. They perform classification on emotional valence using a discrete 5-point scale and 3-point scale and achieve an accuracy 
of $43.88 \%$ and $49.80 \%$, respectively. Sahu et al. [154] use a conditional GAN for modelling feature representations and generating new data samples. They performed evaluations on the IEMOCAP and MSP-IMPROV datasets and showed that synthetic feature vectors can help improve SER performance in different settings. In another study [155], the authors use a GAN to generate a high dimensional synthetic feature representation using lower-dimensional feature vectors and apply synthetic feature representations to augment the training data. Based on the within-corpus and cross-corpus evaluations, they find that synthetic data can help to improve performance. In [156], the authors present a semi-supervised adversarial model to facilitate knowledge transfer from videos to the audio domain, hence, improving SER performance. They show that the proposed model can outperform a baseline supervised method on the CREMA-D and RAVDESS datasets. Zhao et al. [157] present robust semi-supervised GANs to address the issue of labelled data unavailability. They evaluate the model on four publicly available datasets for capturing underlying emotional representation knowledge from both labelled and unlabelled data. They demonstrate that the proposed methods are superior to the state-of-the-art supervised and semi-supervised models. Sahu et al. [158] evaluate semisupervised AAEs in SER for encoding emotional representation in a compressed form and generating the synthetic data samples. They perform experiments on IEMOCAP and observed that an AAE can encode emotional representation in compressed form without losing emotional class discriminability and can generate synthetic samples that augment the training data to improve the SER performance.

Semi-supervised representation learning is mainly used in SER to circumvent the lack of sufficient labelled training data by utilising unlabelled data. These studies show that semi-supervised representation learning helps to learn generalised representations by including the unlabelled data into the training pipeline, which leads to performance improvements. Another way of using unlabelled data is the representation transfer learning that we discuss next.

\subsection{Representation Transfer Learning}

Transfer learning (TL) involves methods that utilise any knowledge resources (i.e., data, model, representations, labels, etc.) to increase the model learning and generalisation for the target task [159]. The idea behind TL is "Learning to Learn", which specifies that learning from scratch (tabula rasa learning) is often limited, and experience should be used for deeper understanding [160]. Representation transfer learning involves using representations learnt on any large scale data and can be beneficially utilised for the target task. It encompasses different approaches, including multitask learning (MTL), domain adaptation, knowledge transfer, covariance, self-supervised learning, etc. This subsection covers domain adaptive, multitask, and self-supervised representation learning, which is a very popular representation transfer learning approach to improve SER's performance.

\subsubsection{Deep Domain Adaptive Representation Learning}

Deep domain adaptive representation learning is a subfield of TL, and it has emerged to address the problem of domain shift. SER systems can achieve better results when evaluated on test data having a distribution similar to the training set. However, the performance of SER systems is degraded by the mismatch in training and testing data distributions. These differences become more significant with the training and test data of different languages, leading to failing SER systems to function. To build more robust systems for SER applications, domain adaptive representation learning techniques are usually applied to explicitly minimise the difference between the training (source) and testing (target) domains.

In SER, various domain adaptive representation learning methods are evaluated to enable the system to learn representations that can be used to perform emotion identification across different corpora and different languages. Deng et al. [161] use a DAE with shared hidden layers to learn common representations for different emotional datasets. The proposed model can minimise the discrepancy between different datasets and increase the emotion classification accuracy compared to other feature domain adaptation methods. In [162], the authors introduce shared hiddenlayer AE to learn common feature representations shared across the source and target data to reduce the discrepancy in them. They perform evaluations on three publicly available corpora and demonstrate that the proposed method significantly improves the emotion classification accuracy compared to a DAE. In another study [115], the authors use a Universum AE for unsupervised domain adaptation to improve cross-corpus SER. They performed evaluations on four publicly available datasets and showed that the Universum AE has the strong representational capability to discover common structures among the source and target speech data.

Domain adversarial neural networks (DANNs) [163] are becoming popular in learning domain adaptive representation learning. The authors in [117] suggest an adversarial domain network for cross-corpus emotional attributes' prediction. They focus on capturing common representations between the train and test domains by applying a gradient reversal layer (GRL) which propagates back the gradient produced by the domain classifier to the shared layers. They observe that the proposed model can learn domain invariant representations to improve the primary regression task. Xiao et al. [164] present an adversarial network for class-Aligned and generalised domain-invariant representations learning. They also consider GRL to facilitate shared representations among source and target domains. They evaluate the proposed architecture against cross-corpus settings and achieve improved results compared to AE-based models and Danns. In [165], the authors evaluate a DANN against cross-lingual SER. They use GRL with a language classifier, which helps the model to learn language-independent emotional representations. Experiments with the IEMOCAP and RECOLA datasets show that their proposed method achieves 3.91\% improved accuracy than the baseline system (naive cross-lingual SER) for the arousal and valence classification tasks.

Some studies also use different adversarial networks for domain adaptive representation learning for cross-corpus and cross-language SER. Zhou et al. [166] investigate a class-wise domain adaptation method using adversarial training to address cross-corpus mismatch issues and show 
that adversarial training is useful when the model is to be trained on a target language with minimal labels. The authors perform evaluations on the EMODB, and FAUAIBO datasets [167] and show that the proposed architecture learns generalised representations that minimise the domain shift between positive and negative emotion classes. Gideon et al. [168] introduce an adversarial discriminative domain generalisation method that follows a "meet in the middle" approach for cross-corpus emotion recognition. The proposed model improves the cross-corpus generalisation by iteratively moving the learnt representations for each dataset closer. They perform evaluations on the IEMOCAP, MSP-IMPROV, and PRIORI emotion datasets [169] and find that the proposed model consistently converges and generates more generalised representations for cross-corpus SER, even when no target labelled data is used. In [20], the authors utilise a GAN-based model in an unsupervised way to learn language invariant features and evaluate the model over four different language datasets. They significantly improve the performance of SER across different languages using language invariant representations.

\subsubsection{Multi-Task Representation Learning}

Multi-task learning (MTL) has led to successes in different applications of ML, from NLP [170] and speech analysis [171] to computer vision [172]. MTL aims to optimise more than one loss function in contrast to single-task learning (STL) and uses auxiliary tasks to improve the main task of interest [173]. Multitask representation learning (MTRL) can improve the performance of the main task by capturing underlying relevant factors from the auxiliary tasks [9], [174]. In this way, representations learnt in the MTL scenario become more generalised, which helps improve the performance. In SER, the speech also contains multi-dimensional information about the message, speaker, and gender that can be used as auxiliary tasks to improve performance without external speech data.

For SER, studies use emotional attributes (e.g., arousal and valance) as auxiliary tasks to improve the performance of the system. Xia et al. [111] apply a DBN-based MTL model that uses dimensional emotions as auxiliary tasks to improve the performance of categorical emotion as a major task. Their results indicate that learning shared representations for different tasks acts as complementary information to SER systems and helps to improve performance. In [175], Kim et al. presented an MTRL framework that utilises gender and naturalness as auxiliary tasks. They evaluate the proposed model with within-corpus and cross-corpus settings on five publicly available datasets and find that MTL improves the generalisation in SER by learning more generalised representations when compared to the stateof-the-art STL methods. Further, Parthasarathy et al. [176] proposed an MTRL framework for joint prediction of emotional attributes including arousal, valence, and dominance by exploiting their interdependencies. Their results indicate that the proposed model learns shared representations that maximise the performance of the regression models. They base their experiments on three datasets for evaluations and demonstrate that the proposed MTRL model gains a concordance correlation coefficient (CCC) as high as $4.7 \%$ for within-corpus and $14.0 \%$ for cross-corpora experiments compared to STL. In [112], the authors introduce an MTRL framework for jointly learning primary and secondary emotions. They perform evaluations on the MSP-Podcast database and show that the proposed MTRL model can leverage the extra information about the secondary emotions and leads to relative improvements of 7.9\% in F1-score for an 8-class emotion classification task.

Other auxiliary tasks that researchers consider in MTL SER are speaker and gender recognition to improve the accuracy of a system compared to STL [177]. In [113], Nediyanchath et al. utilise a multi-head attention-based MTRL framework with gender classification as an auxiliary information source. They find that gender-specific representations influence the emotion characteristics in speech and achieved $70.1 \%$ for UA-that is $5.3 \%$ higher than the stateof-the-art reported accuracy in SER for four emotion classes at the time. Tao et al. [114] utilise a variant of a multitask LSTM for learning contextual representations with speaker and gender as auxiliary tasks. The proposed model learns a shared representation for multitasks, which help to achieve a 5.5\% relatively higher accuracy than the 'standard' LSTM on the IEMOCAP dataset. Next, Latif et al. [109] introduce an MTRL framework that uses auxiliary tasks for which data is abundantly available and find that utilisation of this additional data for auxiliary tasks can improve the main task of emotion classification with limited available labelled data. They apply AAE to learn powerful and discriminative representations with gender identification and speaker recognition as the auxiliary tasks. Evaluations performed on IEMOCAP, and MSP-IMPROV show that the proposed model can generate generalised and discriminative representations that help to achieve results better than the stateof-the-art comparable studies, with a supervised single- and multitask CNN, and single- and multitask semi-supervised AEs.

MTRL is an effective approach to learning a shared representation that leads to no major increase in computational power while improving the system's recognition accuracy and decreasing the chance of overfitting [109]. However, MTL implies the preparation of labels for considered auxiliary tasks, which is expensive and time-consuming. Recently, self-supervised representation learning is emerging as a solution to utilise representations learnt from unlabelled data to supervise a downstream task. We discuss selfsupervised representation learning next.

\subsubsection{Self-Supervised Representation Learning}

Self-supervised representation learning [178] is a new paradigm in ML, which is a form of unsupervised learning, where the data provides the supervision. The selfsupervised task, also known as the pretext task, uses the unlabelled data to guide downstream tasks. Self-supervised representation learning utilises both labelled and unlabelled data. However, unlabelled data do not need to belong to the same class labels or generative distribution as the labelled data. Such a loose restriction on unlabelled data in selfsupervised learning significantly simplifies learning from a large volume of unlabelled data.

Self-supervised representation learning-based models are getting tremendous interest in vision [179], NLP [180], and speech recognition [181], however, emotional represen- 
tation learning with self-supervising needs exploration in SER. We find a recent study [182] that presents a visual dataguided self-supervised framework for speech representation learning. The authors evaluate the proposed model in SER and automatic speech recognition (ASR) and achieve stateof-the-art results for emotion recognition and competitive results for speech recognition. In [183], the authors propose a multitask self-supervised method for shared speech representation learning, where a single neural encoder is followed by multiple workers that jointly solve different self-supervised tasks. They achieve improved results for speaker, phonemes, and emotional cues identification. Further, transformers [184] are becoming very popular in applying a self-supervised multi-modal representation to improve SER [185]. In [186], the authors exploit transformer-based self-supervised representation learning to improve multimodal emotion recognition. They report that fine-tuning the transformer from the masked language modelling task improve emotion recognition performance by $3 \%$ on the CMU-MOSE dataset. These studies indicate the potential of self-supervised representation learning, which need to be further explored in SER.

\subsection{DRL for Representation Learning}

Deep reinforcement learning (DRL) is a combination of DL and reinforcement learning (RL) principles to create efficient and autonomous systems that can learn by interacting with their environment. RL follows the principle of behaviourist psychology, where an agent learns to take actions in an environment and tries to maximise the accumulated reward over its lifetime. RL has been repeatedly successful in solving various problems [187]; however, previous methods were inherently limited to low-dimensional problems and lacked scalability. The advancements in DL have accelerated the progress in RL and gave rise to various algorithms to solve high-dimensional complex problems [188].

Recently, DRL is also gaining interest in the speech community, and researchers have proposed multiple approaches to model different speech problems [189]. Some of the popular RL-based solutions include dialog modelling and optimisation [190], [191], speech recognition [192], and speech enhancement [193]. In SER, researchers also use DRL algorithms for emotion modelling in speech [121], [194]. However, the problem of emotional representation learning for improving SER is not explored using DRL.

\section{Challenges of Deep Representation LEARNING IN SER}

In this section, we present the major challenges of using deep representation learning in SER.

\subsection{Training Complexity}

Training DL models for representation learning is not straightforward. It applies highly non-linear functions to the input signal to learn abstract representations. Learning representations associated with input manifolds requires intense and difficult training to unfold and distort complicated input manifolds [9]. Speech signals have complex manifolds [195] that inherently embody information related to the message as well as the speaker's gender, age, health status, personality, friendliness, mood, and emotion. These types of information are entangled together [40], and training DL models for disentanglement of emotional representations from other attributes (in a latent space) is a difficult task and has been a long-standing goal in SER.

The training of unsupervised representation learning models in SER is much more difficult than that of supervised ones. Due to relatively small emotional speech datasets, unsupervised representation learning methods do not guarantee to learn a useful representation and can potentially ignore emotional attributes [196]. In contrast, unsupervised representation learning from larger audio or multi-modal datasets can be potentially used to improve the SER performance. However, the performance improvement is not significant despite increasing the complexity of the system by using hundreds of hours of audio data [146]. Therefore, semi-supervised representation learning models are considered as an alternative, as they utilise both labelled and unlabelled data (as discussed in Section 3.3). However, blind training of DL models for semi-supervised representation learning may not necessarily improve the performance over supervised learning [197]. Empirical evidence suggests that unlabelled data only help in certain favourable situations when there is a link between the marginal data distribution and the target function [198]. In fact, noisy and biased unlabelled data can even lead to worse performance [199]. Therefore, it is required to manually select the learning parameters and regularise the semi-supervised model to learn a generalised representation from both, labelled and unlabelled data that help improve the performance compared to the supervised techniques.

Literature shows that GANs have a strong ability to model data distribution and learn discriminative representations. However, they are difficult to train on available emotional corpora, as they face convergence issues [155]. To address this, researchers use various techniques, including conditional architectures [155], [200], unlabelled data [201], and data augmentation techniques [103] with substantial room for further improvement for effective training of GANs in learning emotion representations.

\subsection{Lack of Emotional Speech Data}

Deep representation learning models aim to identify potentially useful and ultimately understandable patterns. This demands not just plenty of data, but diverse data that capture all the directions of variation in the data [202]. For learning a good representation, data must be accurately labelled and unbiased. Most of the SER corpora are designed in laboratories, which may have bias and the recorded corpus on acted emotions may not represent real-life human emotions. This can lead the algorithms to exhibit erroneous behaviour [203].

The quality of speech emotional data can also be poor due to various other reasons. For example, different background noises and music can corrupt speech data. Similarly, the noise of microphones or recording devices can also contaminate the speech signal. Although studies use 'noise injection' techniques to avoid overfitting, this only works for moderately high signal-to-noise ratios [204]. Due to the current emphasis on emotion recognition in the wild, this has become ever so important to recognise speech emotion from noisy data. DAEs [63] can learn a representation of 
data with noise, imputation AE [205] can learn a representation from incomplete data, and non-local AE [206] can learn reliable representations from corrupted data. These models are very popular for noise invariant emotional representation learning; however, their performance still needs further improvement.

In SER, the design and use of the existing emotional speech database primarily depend on the research goals. For example, the emotions can be classified as soothing, and prohibition [207]; or joy and anger [208]. The number and type of emotions contained in a database define the emotional classification task. In most cases, the corpora are purpose-driven, developed by professional actors, and do not naturally incorporate and simulate emotions. Most importantly, these corpora are annotated by human raters as an 'outer emotion', which can be highly different from the 'inner emotion' of an individual. Representations learnt from such laboratory designed datasets cannot be generalised to real-life natural emotions. To eventuate real-world applications of deep emotional representation learning-based SER systems, there is still a need for generic emotional speech corpora by using standard ground truth, which captures all the human emotions. Moreover, these emotional speech databases should be standardised and available for the research community.

\subsection{Corpus and Lingual Variance}

Deep representation learning-based SER systems have achieved improved results when evaluated using similar training and testing data. However, the performance of these systems drops significantly if the test samples deviate from the distribution of the training data. Learning emotional representations that are invariant to speakers, language, etc., are difficult to achieve. The representations learnt from one corpus tend not to work well on other corpora with different recording conditions.

In the past few years, researchers have achieved competitive performance by learning speaker invariant emotional representations [209], [210]. However, language and corpus invariant representation learning is still a very challenging task. Although emotions are considered language invariant, the performance of SER systems degrades when tested across different language emotional corpora [20]. Representations learnt by a few shot learning can be a solution for adapting SER systems, which needs a few samples of target language data. Compared to the number of spoken languages globally, we have speech corpora covering a few languages only. Even though there are more than 5000 spoken languages in the world, 389 languages alone account for $94 \%$ of the world's population ${ }^{2}$. However, speech corpora are missing even for all of these 389 languages, which makes cross-language speech emotion research more challenging. The variation, imbalance, diversity, and dynamics in speech and language corpora present hurdles to designing generalised representation learning algorithms. Recent studies are focusing on representation learning for languages with the very small size of emotional datasets [20], but a fully satisfactory solution has not yet emerged.

2. https://www.ethnologue.com/statistics

\subsection{Privacy and Robustness Issues}

When people use SER services, they usually grant complete access to their speech recordings or transmit features through the network. It can cause a leak of user's information such as gender, ethnicity, and emotional state and can be used for unintended purposes [40], [211]. Similarly, the users' recordings can also be edited or used to create a fake speech that the user never spoke, or the voiceprints can be used to deceive voice-authentication systems. In healthcare applications of SER, there are also risks of users' personal private information leakage [212]. Various other privacyrelated issues that arise while using speech-based services have been discussed in [213]. It is desirable in SER applications that there are suitable provisions for ensuring that there is no unauthorised and undisclosed eavesdropping and violation of privacy.

Privacy-preserved representation learning can alleviate this problem but is a relatively unexplored research topic. Recently, researchers have started to utilise privacy-preserving representation learning models to protect speaker identity [214], gender identity [211], and language information [40]. This motivates the exploitation of deep representation learning models on devices, or edge servers [212]. In this way, robust representation from speech in smaller dimensions can be learned and transmitted to the network for real-life applications [40]. However, model or feature sizes should be optimised for on-device utilisation for feature extraction. To preserve users' privacy, federated learning [215] is another technique, explored in [216] for SER, where the training of a shared global model is performed using multiple participating computing devices. These participating devices collaboratively learn a shared model without revealing their local data and avoid privacy infringement.

Recent studies on adversarial examples pose enormous challenges for robust representation learning from speech by showing the susceptibility of deep models to adversarial examples having imperceptible perturbations [217]. Some popular adversarial attacks include the fast gradient sign method (FGSM) [218], Jacobian-based saliency map attack (JSMA) [219], and DeepFool [220]. They compute the perturbation noise based on the gradient of the targeted output. SER systems are also vulnerable to these attacks [131], [221]. The success of adversarial attacks against SER systems shows that the representations learnt by underlying DL models are not robust [222]. Immunity against such adversarial perturbations, which could mislead SER classifiers, can be achieved by training a DL model to generate an invariant representation to such transformations. This has been explored in the image domain [223] but needs to be explored for emotional representation learning from speech. In SER, emotional representations learnt by very deep architectures are found robust against adversarial attacks [131]. However, further research is required to tackle the challenge of adversarial attacks by exploring what DL models capture from the input speech data and how adversarial examples can be defined as a combination of previously learnt representations without any knowledge of adversaries [224]. 
TABLE 6: Summary of challenges, gaps, and future directions of deep representation learning in SER.

\begin{tabular}{|l|l|l|l|}
\hline Challenges & $\begin{array}{l}\text { Solutions explored } \\
\text { in Literature }\end{array}$ & Existing Gaps & Future Directions \\
\hline Training complexity & $\begin{array}{l}\text { Static representation } \\
\text { learning methods }\end{array}$ & Lack of exploration & $\begin{array}{l}\text { DRL-based methods need to be explored } \\
\text { for emotional representation learning }\end{array}$ \\
\hline $\begin{array}{l}\text { Limited size } \\
\text { labelled emotional data. }\end{array}$ & $\begin{array}{l}\text { Unlabelled data using } \\
\text { Unsupervised representation } \\
\text { learning techniques. }\end{array}$ & Low performance. & $\begin{array}{l}\text { Investigation of self-supervised } \\
\text { representation learning methods. }\end{array}$ \\
\hline $\begin{array}{l}\text { Corpus and } \\
\text { Lingual Variance }\end{array}$ & $\begin{array}{l}\text { Domain adaptive } \\
\text { representation learning } \\
\text { methods. }\end{array}$ & $\begin{array}{l}\text { Performance not comparable } \\
\text { with baseline results. }\end{array}$ & $\begin{array}{l}\text { Investigation of multi-modal } \\
\text { representation to improve SER. }\end{array}$ \\
\hline $\begin{array}{l}\text { Privacy and Robustness } \\
\text { Issues. }\end{array}$ & $\begin{array}{l}\text { Privacy preserving } \\
\text { representations. }\end{array}$ & $\begin{array}{l}\text { Performance drops using } \\
\text { these representations. }\end{array}$ & $\begin{array}{l}\text { Research is required on effective defence } \\
\text { mechanisms against adversarial attacks. }\end{array}$ \\
\hline
\end{tabular}

\section{Discussion AND FUtURe Directions}

This section highlights the gaps and provides future research pointers for different aspects of deep representation learning in SER. For quick insights, the readers are referred to Table 6 that presents the summary of challenges, solutions presented in the literature, existing gaps, and future directions.

\subsection{Input Features}

In recent years, the trend of using hand-engineered acoustic features has been progressively changing. Deep representation learning is gaining popularity as a viable alternative to learn directly from raw speech or features requiring fewer processing steps. Researchers achieved promising results using $\mathrm{CNNs}$ and $\mathrm{CNN}$-RNNs to learn low-level speech representations from raw waveforms, allowing a network to capture important emotional characteristics better. However, the proper design of the feature extraction block is crucial to achieving this goal [50]. However, raw speech as input to deep models requires enormous data to achieve competitive performance. Researchers use data augmentation techniques to meet the data requirement [50], [225]. Log-Mel features and spectrograms are considered popular choices to alleviate this problem as they need less processing, fewer data samples, and training to achieve state-of-the-art classification performance compared to setups where raw audio is used. Table 5 shows that different hand-engineered features are more popular compared to the raw speech as input. However, it has been shown in recent studies [21], [51] that deep representation learning techniques can extract discriminative representations and a particular choice of input features is not as important as the model architecture. Therefore, future research is required to design deep architectures that have minimal human knowledge to learn generalised representations across emotions, languages, and corpora.

\subsection{Models}

A summary of various deep representation learning techniques is presented in Table 5. Studies using supervised representation learning methods typically focus on learning discriminative and robust representations. Models like CNNs, LSTM/GRU RNNs, and CNN-LSTM/GRU-RNNs are widely used for learning salient emotional representations from raw speech. The reason for their popularity is that CNN layers act as data-driven filterbanks that can model spectral envelope of raw speech, and LSTM/GRURNNs can model contextual information. Therefore, most of the studies on raw speech either use CNN, LSTM/GRURNNs, or their combination for SER. Among these models,
LSTM/GRU-RNN-based architectures are mostly applied due to their ability to capture temporal context. However, RNNs use computationally expensive back-propagation through time (BPTT) [226] to learn temporal dependencies by sequentially processing the speech signal. Recently, Transformers solve this issue by utilising the self-attention mechanism for learning temporal correlations from the sequential data [184]. This makes Transformers capable of capturing more temporal contexts with less computation complexity. Various studies in ASR [227]-[229] and speech synthesis [230], [231] highlight the contextual representation learning ability and computational efficiency of Transformers. Emotions in speech are also contextually dependent. Therefore, Transformers need to be explored in SER.

Following the success of generative models, recent papers are mostly focused on utilising their distribution learning and generation abilities. Models like VAEs, AAAEs, and GANs are becoming popular choices in SER due to their representation learning and feature generating abilities. An interesting utilisation of GANs is generating synthetic data, which can be utilised for SER to solve the data scarcity problem. Learning a deep representation from synthetic data can help improve the performance, and researchers have validated the effective use of synthetic data for SER [103], [158], [201]. However, creating 'accurate' synthetic speech or features in different emotions using the available limited sized emotional corpora is a difficult task, and generative models like GANs face convergence issues [155]. Therefore, further research is needed to explore effective training methods for generative models.

It is still an open research question which deep model is superior for emotional representation learning to improve SER performance. It is very hard to answer this question from this literature search since different studies achieved state-of-the-art results in different settings with different models. This mainly depends on how effectively a particular deep architecture is designed, pre-trained, and tuned for deep representation learning.

\subsection{Training Technique}

We present a comparison in Table 7 on the training techniques of deep representation learning models based on different attributes to highlight trade-offs. Supervised representation learning models are very popular in SER due to better performance. However, the unavailability of labelled data is a real bottleneck. These models require fully labelled data as highlighted in Table 7. Unsupervised representation learning can alleviate this problem by learning emotional structures and patterns without requiring any labels, which can help to improve the performance of the emotion classi- 
TABLE 7: Comparing attributes of different deep representation learning techniques.

\begin{tabular}{|c|c|c|c|c|}
\hline Attributes & $\begin{array}{l}\text { Supervised } \\
\text { Representation } \\
\text { Learning }\end{array}$ & $\begin{array}{l}\text { Unsupervised } \\
\text { Representation } \\
\text { Learning }\end{array}$ & $\begin{array}{l}\text { Semi-Supervised } \\
\text { Representation } \\
\text { Learning } \\
\end{array}$ & $\begin{array}{l}\text { Representation } \\
\text { Transfer } \\
\text { Learning } \\
\end{array}$ \\
\hline Fully labelled Data Compulsory & $\checkmark$ & $x$ & $x$ & $x$ \\
\hline Unlabelled Data & $x$ & $\checkmark$ & $\checkmark$ & $\checkmark$ \\
\hline Partial Labelled Data & $x$ & $x$ & $\checkmark$ & $\checkmark$ \\
\hline Predict Label/Future & $\checkmark$ & $x$ & $\checkmark$ & $\checkmark$ \\
\hline Accuracy & High & Low & High & High \\
\hline Exploration & $x$ & $x$ & $x$ & $x$ \\
\hline
\end{tabular}

fication task [146]. Autoencoding networks are widely used in SER for unsupervised feature learning from speech; however, SER performance with unsupervised models is always inferior to that of supervised models (as highlighted in Table 5 and Table 6). Self-supervised representation learning is gaining interest in vision, NLP, and speech recognition. It enables better utilisation of unlabelled data by learning high-level representations that can be used for different downstream tasks. Research is required to explore the utilisation of representations learnt from multi-modal data by self-supervision on the downstream task of SER.

Semi-supervised representation learning models are widely used in SER because they can exploit both labelled and unlabelled data to learn more. Studies utilise generalised representations from both labelled and unlabelled data to improve the performance of SER. The popular models include GANs [110], AE-based models [109], [151] and other discriminative architectures [232], [233]. However, there are still opportunities in semi-supervised representation learning for improving the performance of SER by concomitantly reducing the annotation burden of emotional corpora. Most importantly, further research is required to provide theoretical guidelines on the number of labelled versus unlabelled samples required to build semisupervised SER systems for practical applications.

MTRL methods are very popular in SER, where researchers utilise additional label information available (e.g., speaker or gender) in speech as auxiliary tasks to learn more generalised representations that help to improve the SER performance [109]. However, extra efforts are required to prepare the labels for the auxiliary task in MTRL approaches, which is an expensive and time-consuming task. Another problem that hinders MTRL is the temporal differences among tasks. For instance, the modelling of speaker recognition requires different temporal information than phoneme recognition [234]. Ideally, memory-based deep neural networks, e. g., LSTM or GRU cells, can help address this issue.

Good representation disentangles the underlying explanatory factors of variation. However, it is an open research question what kind of training framework can potentially learn disentangled representations from input data. As highlighted in Table 7, all static representation learning methods do not involve exploration. Reinforcement learning (RL), on the other hand, facilitates the idea of exploration while learning by interacting with the environment. A good representation can be learned if RL is used to disentangle factors of variation by interacting with the environment. This will lead to faster convergence, in contrast to blindly attempting to solve given problems. Thomas et al. [235] recently validate this idea, where the authors use RL to disentangle the independently controllable factors of variation by using a specific objective function. The authors show that the agent can disentangle these aspects of the environment without any extrinsic reward. This is an important finding that will act as the key to further research in this direction. Some other studies [236]-[239] also use RL-based approaches to learn representations and have achieved considerable success. However, such RL-based approaches need to be further explored for SER.

\section{Conclusions}

This article focuses on providing a comprehensive review of speech emotional representation learning using deep learning approaches. In speech emotion recognition (SER), the use of representation learning is very important, and there is ongoing research on this topic in which different models and methods are being explored to disentangle speech attributes suitable for emotion detection and identification. The highlights of this survey are as follows:

- Most SER corpora are developed in laboratories by professional actors and annotated by human raters as outer emotions that might be significantly different from inner ones. Future efforts are required to motivate the collection of natural emotional corpora by defining standardised data collection protocols with a special focus on protecting speakers' personal information. It will encourage participants to enrol in data collection.

- The SER research community is increasingly shifting its focus to designing systems using raw speech or input features that have minimal human knowledge dependency.

- LSTM/GRU-RNNs combined with CNNs are very popular and suitable for capturing emotional attributes in a supervised way. In unsupervised representation learning, Denoising Autoencoders (DAEs) and Variational Autoencoders (VAEs) are widely deployed architectures in SER, and GAN-based models also gaining attention. Further research on utilising these models in a semi-supervised way is required to learn representation from unlabelled and labelled data.

- Emotions are context-dependent, and Transformers can better capture temporal contexts compared to RNNs. This encourages SER researchers to utilise Transformers in their studies.

- Static deep representation learning methods are very popular; however, they lack exploration. In contrast, Deep Reinforcement Learning (DRL) facilitate exploration by interacting with the environment and learn 
better representation. This calls SER researchers to utilise DRL to disentangle the emotional attributes to achieve better performance.

- Privacy-preserving representation learning in SER is very important to explore. This can help achieve robustness against adversarial attacks and ensure no unauthorised access to users' personal information while using SER services.

- Domain adaptation solutions based on adversarial neural networks are widely used for cross-corpus and cross-language emotion recognition; however, performance is not comparable to baseline. Multimodal self-supervised domain adaptive representation learning models can potentially improve performance.

We believe this article has the potential to become a definitive guide to researchers and practitioners interested in deep representation learning for SER. With all these changes and advancements in place, we look forward to an exciting era of SER starting to enable artificial intelligence (AI) to sense our emotions better. We are curious whether, in the longer run, deep representation learning will be the standard paradigm in SER. If so, we are currently changing a paradigm moving away from signal processing and expertcrafted features into a highly data-driven era-with all its advantages, challenges, and risks.

\section{REFERENCES}

[1] N. Ambady and R. Rosenthal, "Thin slices of expressive behavior as predictors of interpersonal consequences: A meta-analysis," 1992.

[2] S. Kuchibhotla, H. Vankayalapati, R. Vaddi, and K. R. Anne, "A comparative analysis of classifiers in emotion recognition through acoustic features," International Journal of Speech Technology, vol. 17, no. 4, pp. 401-408, 2014.

[3] A. Konar and A. Chakraborty, Emotion recognition: A pattern analysis approach. John Wiley \& Sons, 2014.

[4] Z. Zeng, M. Pantic, G. I. Roisman, and T. S. Huang, "A survey of affect recognition methods: Audio, visual, and spontaneous expressions," IEEE Transactions on Pattern Analysis and Machine Intelligence, vol. 31, no. 1, pp. 39-58, 2009.

[5] Q. Mao, M. Dong, Z. Huang, and Y. Zhan, "Learning salient features for speech emotion recognition using convolutional neural networks," IEEE Transactions on Multimedia, vol. 16, no. 8, pp. 2203-2213, 2014.

[6] C. Huang, W. Gong, W. Fu, and D. Feng, "A research of speech emotion recognition based on deep belief network and SVM," Mathematical Problems in Engineering, vol. 2014, 2014.

[7] Z. Cairong, Z. Xinran, Z. Cheng, and Z. Li, "A novel DBN feature fusion model for cross-corpus speech emotion recognition," Journal of Electrical and Computer Engineering, vol. 2016, 2016.

[8] J. Lee and I. Tashev, "High-level feature representation using recurrent neural network for speech emotion recognition," in INTERSPEECH, 2015, pp. 1537-1540.

[9] Y. Bengio, A. Courville, and P. Vincent, "Representation learning: A review and new perspectives," IEEE Transactions on Pattern Analysis and Machine Intelligence, vol. 35, no. 8, pp. 1798-1828, 2013.

[10] G. Zhong, L.-N. Wang, X. Ling, and J. Dong, "An overview on data representation learning: From traditional feature learning to recent deep learning," The Journal of Finance and Data Science, vol. 2, no. 4, pp. 265-278, 2016.

[11] S. Basu, J. Chakraborty, A. Bag, and M. Aftabuddin, "A review on emotion recognition using speech," in 2017 International Conference on Inventive Communication and Computational Technologies (ICICCT). IEEE, 2017, pp. 109-114.

[12] M. Swain, A. Routray, and P. Kabisatpathy, "Databases, features and classifiers for speech emotion recognition: a review," International Journal of Speech Technology, vol. 21, no. 1, pp. 93-120, 2018.
[13] M. B. Akçay and K. Oğuz, "Speech emotion recognition: Emotional models, databases, features, preprocessing methods, supporting modalities, and classifiers," Speech Communication, vol. 116 , pp. 56-76, 2020.

[14] K. Pearson, "Liii. on lines and planes of closest fit to systems of points in space," The London, Edinburgh, and Dublin Philosophical Magazine and Journal of Science, vol. 2, no. 11, pp. 559-572, 1901.

[15] D. P. Kingma and M. Welling, "Auto-encoding variational bayes," International Conference on Learning Representations (ICLR), 2014.

[16] I. Goodfellow, J. Pouget-Abadie, M. Mirza, B. Xu, D. WardeFarley, S. Ozair, A. Courville, and Y. Bengio, "Generative adversarial nets," in Advances in neural information processing systems, 2014, pp. 2672-2680.

[17] A. Radford, L. Metz, and S. Chintala, "Unsupervised representation learning with deep convolutional generative adversarial networks," International Conference on Learning Representations (ICLR), 2016.

[18] S. Furui, "Speaker-independent isolated word recognition based on emphasized spectral dynamics," in ICASSP'86. IEEE International Conference on Acoustics, Speech, and Signal Processing, vol. 11. IEEE, 1986, pp. 1991-1994.

[19] F. Eyben, K. R. Scherer, B. W. Schuller, J. Sundberg, E. André, C. Busso, L. Y. Devillers, J. Epps, P. Laukka, S. S. Narayanan et al., "The geneva minimalistic acoustic parameter set (gemaps) for voice research and affective computing," IEEE Transactions on Affective Computing, vol. 7, no. 2, pp. 190-202, 2015.

[20] S. Latif, J. Qadir, and M. Bilal, “Unsupervised adversarial domain adaptation for cross-lingual speech emotion recognition," in 2019 8th International Conference on Affective Computing and Intelligent Interaction (ACII). IEEE, 2019, pp. 732-737.

[21] M. Neumann and N. T. Vu, "Attentive convolutional neural network based speech emotion recognition: A study on the impact of input features, signal length, and acted speech," INTERSPEECH, pp. 1263-1267, 2017.

[22] G. Zhong, X. Ling, and L.-N. Wang, "From shallow feature learning to deep learning: Benefits from the width and depth of deep architectures," Wiley Interdisciplinary Reviews: Data Mining and Knowledge Discovery, vol. 9, no. 1, p. e1255, 2019.

[23] R. A. Fisher, "The use of multiple measurements in taxonomic problems," Annals of eugenics, vol. 7, no. 2, pp. 179-188, 1936.

[24] D. R. Hardoon, S. Szedmak, and J. Shawe-Taylor, "Canonical correlation analysis: An overview with application to learning methods," Neural computation, vol. 16, no. 12, pp. 2639-2664, 2004.

[25] I. Borg and P. Groenen, "Modern multidimensional scaling: Theory and applications," Journal of Educational Measurement, vol. 40, no. 3, pp. $277-280,2003$

[26] A. Hyvärinen and E. Oja, "Independent component analysis: algorithms and applications," Neural networks, vol. 13, no. 4-5, pp. 411-430, 2000.

[27] F. Lee, R. Scherer, R. Leeb, A. Schlögl, H. Bischof, and G. Pfurtscheller, Feature mapping using PCA, locally linear embedding and isometric feature mapping for EEG-based brain computer interface. Citeseer, 2004.

[28] S. T. Roweis and L. K. Saul, "Nonlinear dimensionality reduction by locally linear embedding," science, vol. 290, no. 5500, pp. 2323 2326, 2000.

[29] T. Virtanen, B. Raj, J. F. Gemmeke et al., "Active-set newton algorithm for non-negative sparse coding of audio," in 2014 IEEE International Conference on Acoustics, Speech and Signal Processing (ICASSP). IEEE, 2014, pp. 3092-3096.

[30] J. B. Tenenbaum, V. De Silva, and J. C. Langford, "A global geometric framework for nonlinear dimensionality reduction," science, vol. 290, no. 5500, pp. 2319-2323, 2000.

[31] L. v. d. Maaten and G. Hinton, "Visualizing data using t-sne," Journal of machine learning research, vol. 9, no. Nov, pp. 2579-2605, 2008.

[32] Y. LeCun, L. Bottou, Y. Bengio, P. Haffner et al., "Gradient-based learning applied to document recognition," Proceedings of the IEEE, vol. 86, no. 11, pp. 2278-2324, 1998.

[33] B. Schölkopf, A. Smola, and K.-R. Müller, "Nonlinear component analysis as a kernel eigenvalue problem," Neural computation, vol. 10, no. 5, pp. 1299-1319, 1998.

[34] G. Baudat and F. Anouar, "Generalized discriminant analysis using a kernel approach," Neural computation, vol. 12, no. 10, pp. 2385-2404, 2000.

[35] M. Längkvist, L. Karlsson, and A. Loutfi, "A review of unsupervised feature learning and deep learning for time-series modeling," Pattern Recognition Letters, vol. 42, pp. 11-24, 2014. 
[36] G. E. Hinton and R. R. Salakhutdinov, "Reducing the dimensionality of data with neural networks," science, vol. 313, no. 5786, pp. 504-507, 2006

[37] Y. Bengio, P. Lamblin, D. Popovici, and H. Larochelle, "Greedy layer-wise training of deep networks," in Advances in neural information processing systems, 2007, pp. 153-160.

[38] C. Poultney, S. Chopra, Y. L. Cun et al., "Efficient learning of sparse representations with an energy-based model," in Advances in neural information processing systems, 2007, pp. 1137-1144.

[39] I. Goodfellow, Y. Bengio, and A. Courville, Deep Learning. MIT Press, 2016, http:/ / www.deeplearningbook.org.

[40] H. S. Ali, F. ul Hassan, S. Latif, H. U. Manzoor, and J. Qadir "Privacy enhanced speech emotion communication using deep learning aided edge computing," in 2021 IEEE International Conference on Communications Workshops (ICC Workshops). IEEE, 2021, pp. $1-5$.

[41] D. O. Hebb, The organization of behavior: A neuropsychological theory. Psychology Press, 1949.

[42] F. Rosenblatt, "The perceptron: a probabilistic model for information storage and organization in the brain." Psychological review, vol. 65 , no. 6 , p. 386,1958

[43] Y. Bengio et al., "Learning deep architectures for AI," Foundations and trends ${ }^{\circledR}$ in Machine Learning, vol. 2, no. 1, pp. 1-127, 2009.

[44] R. K. Srivastava, K. Greff, and J. Schmidhuber, "Training very deep networks," in Proceedings of the 28th International Conference on Neural Information Processing Systems-Volume 2, 2015, pp. 23772385

[45] Y. LeCun, B. Boser, J. Denker, D. Henderson, R. Howard, W. Hubbard, and L. Jackel, "Handwritten digit recognition with a backpropagation network," Advances in neural information processing systems, vol. 2, 1989.

[46] I. Goodfellow, Y. Bengio, and A. Courville, Deep learning. MIT press, 2016.

[47] K. He, X. Zhang, S. Ren, and J. Sun, "Deep residual learning for image recognition," in Proceedings of the IEEE Conference on Computer Vision and Pattern Recognition, 2016, pp. 770-778.

[48] G. Huang, Z. Liu, L. Van Der Maaten, and K. Q. Weinberger, "Densely connected convolutional networks," in Proceedings of the IEEE Conference on Computer Vision and Pattern Recognition, 2017, pp. 4700-4708.

[49] Y. LeCun, Y. Bengio et al., "Convolutional networks for images, speech, and time series," The handbook of brain theory and neural networks, vol. 3361, no. 10, p. 1995, 1995.

[50] S. Latif, R. Rana, S. Khalifa, R. Jurdak, and J. Epps, "Direct modelling of speech emotion from raw speech," INTERSPEECH, pp. 3920-3924, 2019.

[51] Z. Aldeneh and E. M. Provost, “Using regional saliency for speech emotion recognition," in 2017 IEEE International Conference on Acoustics, Speech and Signal Processing (ICASSP). IEEE, 2017, pp. 2741-2745.

[52] D. E. Rumelhart, G. E. Hinton, and R. J. Williams, "Learning representations by back-propagating errors," nature, vol. 323, no. 6088, pp. 533-536, 1986.

[53] M. I. Jordan, "Serial order: A parallel distributed processing approach," in Advances in psychology. Elsevier, 1997, vol. 121, pp. 471-495.

[54] K. Cho, B. van Merrienboer, C. Gulcehre, F. Bougares, H. Schwenk, and Y. Bengio, "Learning phrase representations using RNN encoder-decoder for statistical machine translation," in Conference on Empirical Methods in Natural Language Processing (EMNLP 2014), 2014

[55] S. Hochreiter and J. Schmidhuber, "Long short-term memory," Neural computation, vol. 9, no. 8, pp. 1735-1780, 1997.

[56] M. Schuster and K. K. Paliwal, "Bidirectional recurrent neural networks," IEEE Transactions on Signal Processing, vol. 45, no. 11, pp. 2673-2681, 1997.

[57] A. Graves, S. Fernández, F. Gomez, and J. Schmidhuber, "Connectionist temporal classification: labelling unsegmented sequence data with recurrent neural networks," in Proceedings of the $23 \mathrm{rd}$ international conference on Machine learning, 2006, pp. 369-376.

[58] G. E. Hinton and R. S. Zemel, "Autoencoders, minimum description length and helmholtz free energy," in Advances in neural information processing systems, 1994, pp. 3-10.

[59] M. Usman, S. Latif, and J. Qadir, "Using deep autoencoders for facial expression recognition," in 2017 13th International Conference on Emerging Technologies (ICET). IEEE, 2017, pp. 1-6.

[60] A. Ng et al., "Sparse autoencoder," CS294A Lecture notes, vol. 72, no. 2011, pp. 1-19, 2011.
[61] A. Makhzani and B. Frey, "K-sparse autoencoders," arXiv preprint arXiv:1312.5663, 2013

[62] J. Deng, Z. Zhang, E. Marchi, and B. Schuller, "Sparse autoencoder-based feature transfer learning for speech emotion recognition," in 2013 Humaine Association Conference on Affective Computing and Intelligent Interaction. IEEE, 2013, pp. 511-516.

[63] P. Vincent, H. Larochelle, Y. Bengio, and P.-A. Manzagol, "Extracting and composing robust features with denoising autoencoders," in Proceedings of the 25th international conference on $\mathrm{Ma}$ chine learning. ACM, 2008, pp. 1096-1103.

[64] R. Xia and Y. Liu, "Using denoising autoencoder for emotion recognition," in INTERSPEECH, 2013, pp. 2886-2889.

[65] G. E. Hinton, S. Osindero, and Y.-W. Teh, "A fast learning algorithm for deep belief nets," Neural computation, vol. 18, no. 7 , pp. 1527-1554, 2006

[66] D. H. Ackley, G. E. Hinton, and T. J. Sejnowski, "A learning algorithm for Boltzmann machines," Cognitive science, vol. 9, no. 1, pp. 147-169, 1985

[67] Y. Bengio, E. Laufer, G. Alain, and J. Yosinski, “Deep generative stochastic networks trainable by backprop," in International Conference on Machine Learning, 2014, pp. 226-234.

[68] S. Latif, R. Rana, J. Qadir, and J. Epps, “Variational autoencoders for learning latent representations of speech emotion: A preliminary study," INTERSPEECH, pp. 3107-3111, 2018.

[69] I. Higgins, L. Matthey, A. Pal, C. Burgess, X. Glorot, M. Botvinick, S. Mohamed, and A. Lerchner, "beta-VAE: Learning basic visual concepts with a constrained variational framework," ICLR, vol. 2, no. 5, p. 6, 2017.

[70] S. Zhao, J. Song, and S. Ermon, "InfoVAE: Balancing learning and inference in variational autoencoders," Proceedings of the AAAI Conference on Artificial Intelligence, vol. 33, pp. 5885-5892, Jul. 2019.

[71] H. Kim and A. Mnih, "Disentangling by factorising," in International Conference on Machine Learning. PMLR, 2018, pp. 26492658.

[72] M. Tschannen, O. Bachem, and M. Lucic, "Recent advances in autoencoder-based representation learning," Third workshop on Bayesian Deep Learning (NeurIPS), 2018.

[73] C. M. Lee and S. S. Narayanan, "Toward detecting emotions in spoken dialogs," IEEE Transactions on Speech and Audio Processing, vol. 13, no. 2, pp. 293-303, 2005.

[74] D. Ververidis and C. Kotropoulos, "A state of the art review on emotional speech databases," in Proceedings of 1st Richmedia Conference. Citeseer, 2003, pp. 109-119.

[75] M. El Ayadi, M. S. Kamel, and F. Karray, "Survey on speech emotion recognition: Features, classification schemes, and databases," Pattern Recognition, vol. 44, no. 3, pp. 572-587, 2011.

[76] P. Ekman, "Expression and the nature of emotion," Approaches to emotion, vol. 3, pp. 19-344, 1984.

[77] J. A. Russell, "A circumplex model of affect." Journal of personality and social psychology, vol. 39, no. 6, p. 1161, 1980.

[78] F. Burkhardt, A. Paeschke, M. Rolfes, W. F. Sendlmeier, and B. Weiss, "A database of German emotional speech," in INTERSPEECH, vol. 5, 2005, pp. 1517-1520.

[79] C. Busso, S. Parthasarathy, A. Burmania, M. AbdelWahab, N. Sadoughi, and E. M. Provost, "MSP-IMPROV: An acted corpus of dyadic interactions to study emotion perception," IEEE Transactions on Affective Computing, vol. 8, no. 1, pp. 67-80, 2016.

[80] R. Lotfian and C. Busso, "Building naturalistic emotionally balanced speech corpus by retrieving emotional speech from existing podcast recordings," IEEE Transactions on Affective Computing, vol. 10, no. 4, pp. 471-483, 2017.

[81] G. McKeown, M. Valstar, R. Cowie, M. Pantic, and M. Schroder "The semaine database: Annotated multimodal records of emotionally colored conversations between a person and a limited agent," IEEE Transactions on Affective Computing, vol. 3, no. 1, pp. 5-17, 2012.

[82] C. Busso, M. Bulut, C.-C. Lee, A. Kazemzadeh, E. Mower, S. Kim J. N. Chang, S. Lee, and S. S. Narayanan, "IEMOCAP: Interactive emotional dyadic motion capture database," Language resources and evaluation, vol. 42, no. 4, p. 335, 2008.

[83] G. Costantini, I. Iaderola, A. Paoloni, and M. Todisco, "EMOVO corpus: an Italian emotional speech database," in International Conference on Language Resources and Evaluation (LREC 2014). European Language Resources Association (ELRA), 2014, pp. 3501-3504.

[84] F. Ringeval, A. Sonderegger, J. Sauer, and D. Lalanne, "Introducing the RECOLA multimodal corpus of remote collaborative and 
affective interactions," in 2013 10th IEEE international conference and workshops on automatic face and gesture recognition (FG). IEEE, 2013, pp. 1-8.

[85] A. B. Zadeh, P. P. Liang, S. Poria, E. Cambria, and L.-P. Morency, "Multimodal language analysis in the wild: Cmu-mosei dataset and interpretable dynamic fusion graph," in Proceedings of the 56th Annual Meeting of the Association for Computational Linguistics (Volume 1: Long Papers), 2018, pp. 2236-2246.

[86] B. Schuller, A. Batliner, S. Steidl, and D. Seppi, "Recognising Realistic Emotions and Affect in Speech: State of the Art and Lessons Learnt from the First Challenge," Speech Communication, vol. 53, no. 9/10, pp. 1062-1087, November/December 2011.

[87] P. Tzirakis, J. Zhang, and B. W. Schuller, "End-to-end speech emotion recognition using deep neural networks," in 2018 IEEE International Conference on Acoustics, Speech and Signal Processing (ICASSP). IEEE, 2018, pp. 5089-5093.

[88] Y. Huang, K. Tian, A. Wu, and G. Zhang, "Feature fusion methods research based on deep belief networks for speech emotion recognition under noise condition," Journal of Ambient Intelligence and Humanized Computing, vol. 10, no. 5, pp. 1787-1798, 2019.

[89] S. Latif, R. Rana, S. Younis, J. Qadir, and J. Epps, "Transfer learning for improving speech emotion classification accuracy," INTERSPEECH, pp. 257-261, 2018.

[90] K. Han, D. Yu, and I. Tashev, "Speech emotion recognition using deep neural network and extreme learning machine," in Fifteenth annual conference of the international speech communication association, 2014.

[91] A. Stuhlsatz, C. Meyer, F. Eyben, T. Zielke, G. Meier, and B. Schuller, "Deep neural networks for acoustic emotion recognition: Raising the benchmarks," in 2011 IEEE international conference on acoustics, speech and signal processing (ICASSP). IEEE, 2011, pp. 5688-5691.

[92] L. Sun, B. Zou, S. Fu, J. Chen, and F. Wang, "Speech emotion recognition based on DNN-decision tree SVM model," Speech Communication, vol. 115, pp. 29-37, 2019.

[93] H. M. Fayek, M. Lech, and L. Cavedon, "Evaluating deep learning architectures for speech emotion recognition," Neural Networks, vol. 92, pp. 60-68, 2017.

[94] E. Kim and J. W. Shin, "DNN-based emotion recognition based on bottleneck acoustic features and lexical features," in IEEE International Conference on Acoustics, Speech and Signal Processing (ICASSP). IEEE, 2019, pp. 6720-6724.

[95] B. Schuller, D. Arsic, G. Rigoll, M. Wimmer, and B. Radig, "Audiovisual behavior modeling by combined feature spaces," in 2007 IEEE International Conference on Acoustics, Speech and Signal Processing-ICASSP'07, vol. 2. IEEE, 2007, pp. II-733.

[96] D. Bertero and P. Fung, "A first look into a convolutional neural network for speech emotion detection," in 2017 IEEE international conference on acoustics, speech and signal processing (ICASSP). IEEE, 2017, pp. 5115-5119.

[97] D. Bertero, F. B. Siddique, and P. Fung, "Towards a corpus of speech emotion for interactive dialog systems," in 2016 Conference of The Oriental Chapter of International Committee for Coordination and Standardization of Speech Databases and Assessment Techniques (O-COCOSDA). IEEE, 2016, pp. 241-246.

[98] S. Ghosh, E. Laksana, L.-P. Morency, and S. Scherer, "Representation learning for speech emotion recognition," INTERSPEECH, pp. 3603-3607, 2016.

[99] R. Xia, J. Deng, B. Schuller, and Y. Liu, "Modeling gender information for emotion recognition using denoising autoencoder," in 2014 IEEE International Conference on Acoustics, Speech and Signal Processing (ICASSP). IEEE, 2014, pp. 990-994.

[100] B. Schuller, S. Steidl, A. Batliner, F. Burkhardt, L. Devillers, C. Müller, and S. S. Narayanan, "The INTERSPEECH 2010 paralinguistic challenge," in INTERSPEECH, 2010.

[101] G. Paraskevopoulos, E. Tzinis, N. Ellinas, T. Giannakopoulos, and A. Potamianos, "Unsupervised low-rank representations for speech emotion recognition," in INTERSPEECH, 2019, pp. 939943.

[102] S. E. Eskimez, Z. Duan, and W. Heinzelman, "Unsupervised learning approach to feature analysis for automatic speech emotion recognition," in 2018 IEEE International Conference on Acoustics, Speech and Signal Processing (ICASSP). IEEE, 2018, pp. 50995103.

[103] S. Latif, M. Asim, R. Rana, S. Khalifa, R. Jurdak, and B. W. Schuller, "Augmenting generative adversarial networks for speech emotion recognition," INTERSPEECH, pp. 521-525, 2020.
[104] S. Parthasarathy and C. Busso, "Ladder networks for emotion recognition: Using unsupervised auxiliary tasks to improve predictions of emotional attributes," INTERSPEECH, pp. 3698-3702, 2018.

[105] B. Schuller, S. Steidl, A. Batliner, A. Vinciarelli, K. Scherer, F. Ringeval, M. Chetouani, F. Weninger, F. Eyben, E. Marchi et al., "The INTERSPEECH 2013 computational paralinguistics challenge: Social signals, conflict, emotion, autism," in INTERSPEECH, 2013.

[106] S. Parthasarathy and C. Busso, "Semi-supervised speech emotion recognition with ladder networks," IEEE/ACM transactions on audio, speech, and language processing, vol. 28, pp. 2697-2709, 2020.

[107] J.-H. Tao, J. Huang, Y. Li, Z. Lian, and M.-Y. Niu, "Semisupervised ladder networks for speech emotion recognition," International Journal of Automation and Computing, vol. 16, no. 4, pp. 437-448, 2019.

[108] S. Bjorn, S. Steidl, and A. Batliner, "The INTERSPEECH 2009 emotion challenge," in INTERSPEECH), 2009, pp. 312-315.

[109] S. Latif, R. Rana, S. Khalifa, R. Jurdak, J. Epps, and B. W. Schuller, "Multi-task semi-supervised adversarial autoencoding for speech emotion recognition," IEEE Transactions on Affective Computing, 2020.

[110] J. Chang and S. Scherer, "Learning representations of emotional speech with deep convolutional generative adversarial networks," in 2017 IEEE International Conference on Acoustics, Speech and Signal Processing (ICASSP). IEEE, 2017, pp. 2746-2750.

[111] R. Xia and Y. Liu, "A multi-task learning framework for emotion recognition using 2D continuous space," IEEE Transactions on Affective Computing, no. 1, pp. 3-14, 2017.

[112] R. Lotfian and C. Busso, "Predicting categorical emotions by jointly learning primary and secondary emotions through multitask learning," in INTERSPEECH, 2018, pp. 951-955.

[113] A. Nediyanchath, P. Paramasivam, and P. Yenigalla, "Multi-head attention for speech emotion recognition with auxiliary learning of gender recognition," in ICASSP 2020-2020 IEEE International Conference on Acoustics, Speech and Signal Processing (ICASSP). IEEE, 2020, pp. 7179-7183.

[114] F. Tao and G. Liu, "Advanced LSTM: A study about better time dependency modelling in emotion recognition," in 2018 IEEE International Conference on Acoustics, Speech and Signal Processing (ICASSP). IEEE, 2018, pp. 2906-2910.

[115] J. Deng, X. Xu, Z. Zhang, S. Frühholz, and B. Schuller, "Universum autoencoder-based domain adaptation for speech emotion recognition," IEEE Signal Processing Letters, vol. 24, no. 4, pp. 500$504,2017$.

[116] J. Deng, X. Xu, Z. Zhang, S. Frühholz, D. Grandjean, and B. Schuller, "Fisher kernels on phase-based features for speech emotion recognition," in Dialogues with social robots. Springer 2017, pp. 195-203.

[117] M. Abdelwahab and C. Busso, "Domain adversarial for acoustic emotion recognition," IEEE/ACM Transactions on Audio, Speech, and Language Processing, vol. 26, no. 12, pp. 2423-2435, 2018.

[118] A. Shukla, S. Petridis, and M. Pantic, "Does visual selfsupervision improve learning of speech representations for emotion recognition," IEEE Transactions on Affective Computing, 2021.

[119] S. Siriwardhana, T. Kaluarachchi, M. Billinghurst, and S. Nanayakkara, "Multimodal emotion recognition with transformer-based self supervised feature fusion," IEEE Access, vol. 8, pp. 176274-176285, 2020.

[120] S. Schneider, A. Baevski, R. Collobert, and M. Auli, "wav2vec: Unsupervised pre-training for speech recognition," Proc. Interspeech 2019, pp. 3465-3469, 2019.

[121] E. Lakomkin, M. A. Zamani, C. Weber, S. Magg, and S. Wermter, "EMORL: continuous acoustic emotion classification using deep reinforcement learning," in 2018 IEEE International Conference on Robotics and Automation (ICRA). IEEE, 2018, pp. 1-6.

[122] M. Chen, S. Wang, P. P. Liang, T. Baltrušaitis, A. Zadeh, and L.-P. Morency, "Multimodal sentiment analysis with word-level fusion and reinforcement learning," in ACM International Conference on Multimodal Interaction, 2017.

[123] Q. Zhu, M.-C. Yeh, K.-T. Cheng, and S. Avidan, "Fast human detection using a cascade of histograms of oriented gradients," in 2006 IEEE Computer Society Conference on Computer Vision and Pattern Recognition (CVPR'06), vol. 2. IEEE, 2006, pp. 1491-1498.

[124] S. Mirsamadi, E. Barsoum, and C. Zhang, "Automatic speech emotion recognition using recurrent neural networks with local attention," in 2017 IEEE International Conference on Acoustics, Speech and Signal Processing (ICASSP). IEEE, 2017, pp. 2227-2231. 
[125] A. M. Badshah, J. Ahmad, N. Rahim, and S. W. Baik, "Speech emotion recognition from spectrograms with deep convolutional neural network," in International Conference on Platform Technology and Service (PlatCon), 2017. IEEE, 2017, pp. 1-5.

[126] W. Zheng, J. Yu, and Y. Zou, "An experimental study of speech emotion recognition based on deep convolutional neural networks," in 2015 International Conference on Affective Computing and Intelligent Interaction (ACII). IEEE, 2015, pp. 827-831.

[127] J. Liu, W. Han, H. Ruan, X. Chen, D. Jiang, and H. Li, “Learning salient features for speech emotion recognition using CNN," in 2018 First Asian Conference on Affective Computing and Intelligent Interaction (ACII Asia). IEEE, 2018, pp. 1-5.

[128] J. Kim, K. P. Truong, G. Englebienne, and V. Evers, "Learning spectro-temporal features with $3 \mathrm{~d}$ CNNs for speech emotion recognition," in 2017 Seventh International Conference on Affective Computing and Intelligent Interaction (ACII). IEEE, 2017, pp. 383388.

[129] L. Zhu, R. Deng, M. Maire, Z. Deng, G. Mori, and P. Tan, "Sparsely aggregated convolutional networks," in Proceedings of the European Conference on Computer Vision (ECCV), 2018, pp. 186201.

[130] M. Guo, Y. Yang, R. Xu, Z. Liu, and D. Lin, “When NAS meets robustness: In search of robust architectures against adversarial attacks," in Proceedings of the IEEE/CVF Conference on Computer Vision and Pattern Recognition, 2020, pp. 631-640.

[131] S. Latif, R. Rana, S. Khalifa, R. Jurdak, and B. W. Schuller, "Deep Architecture Enhancing Robustness to Noise, Adversarial Attacks, and Cross-Corpus Setting for Speech Emotion Recognition," in INTERSPEECH, 2020, pp. 2327-2331.

[132] A. Triantafyllopoulos, G. Keren, J. Wagner, I. Steiner, and B. W. Schuller, "Towards robust speech emotion recognition using deep residual networks for speech enhancement," in INTERSPEECH, 2019, pp. 1691-1695.

[133] G. Trigeorgis, F. Ringeval, R. Brueckner, E. Marchi, M. A. Nicolaou, B. Schuller, and S. Zafeiriou, "Adieu features? end-to-end speech emotion recognition using a deep convolutional recurrent network," in 2016 IEEE International Conference on Acoustics, Speech and Signal Processing (ICASSP). IEEE, 2016, pp. 5200-5204.

[134] M. Sarma, P. Ghahremani, D. Povey, N. K. Goel, K. K. Sarma, and N. Dehak, "Emotion identification from raw speech signals using DNNs," in INTERSPEECH, 2018, pp. 3097-3101.

[135] X. Wu, S. Liu, Y. Cao, X. Li, J. Yu, D. Dai, X. Ma, S. Hu, $\mathrm{Z}$. Wu, X. Liu et al., "Speech emotion recognition using capsule networks," in ICASSP 2019-2019 IEEE International Conference on Acoustics, Speech and Signal Processing (ICASSP). IEEE, 2019, pp. 6695-6699.

[136] L. Tarantino, P. N. Garner, and A. Lazaridis, "Self-attention for speech emotion recognition," in INTERSPEECH, 2019, pp. 25782582.

[137] S. Yoon, S. Byun, S. Dey, and K. Jung, "Speech emotion recognition using multi-hop attention mechanism," in ICASSP 20192019 IEEE International Conference on Acoustics, Speech and Signal Processing (ICASSP). IEEE, 2019, pp. 2822-2826.

[138] S.-L. Yeh, Y.-S. Lin, and C.-C. Lee, "An interaction-aware attention network for speech emotion recognition in spoken dialogs," in ICASSP 2019-2019 IEEE International Conference on Acoustics, Speech and Signal Processing (ICASSP). IEEE, 2019, pp. 6685-6689.

[139] S. Latif, "Deep representation learning for improving speech emotion recognition," Doctoral Consortium, Interspeech, vol. 2020, 2020.

[140] J. Chorowski, R. J. Weiss, S. Bengio, and A. van den Oord, "Unsupervised speech representation learning using WaveNet autoencoders," IEEE/ACM transactions on audio, speech, and language processing, vol. 27, no. 12, pp. 2041-2053, 2019.

[141] S. Ghosh, E. Laksana, L.-P. Morency, and S. Scherer, "Learning representations of affect from speech," arXiv preprint arXiv:1511.04747, 2015.

[142] Z.-w. Huang, W.-t. Xue, and Q.-r. Mao, "Speech emotion recognition with unsupervised feature learning," Frontiers of Information Technology \& Electronic Engineering, vol. 16, no. 5, pp. 358-366, 2015.

[143] G. Dahl, A.-r. Mohamed, G. E. Hinton et al., "Phone recognition with the mean-covariance restricted Boltzmann machine," in Advances in neural information processing systems, 2010, pp. 469477.

[144] H. B. Sailor and H. A. Patil, "Unsupervised deep auditory model using stack of convolutional rbms for speech recognition," in INTERSPEECH, 2016, pp. 3379-3383.
[145] L. Li, Y. Zhao, D. Jiang, Y. Zhang, F. Wang, I. Gonzalez, E. Valentin, and H. Sahli, "Hybrid deep neural network-hidden Markov model (DNN-HMM) based speech emotion recognition," in 2013 Humaine association conference on affective computing and intelligent interaction. IEEE, 2013, pp. 312-317.

[146] M. Neumann and N. T. Vu, "Improving speech emotion recognition with unsupervised representation learning on unlabeled speech," in ICASSP 2019-2019 IEEE International Conference on Acoustics, Speech and Signal Processing (ICASSP). IEEE, 2019, pp. 7390-7394.

[147] H. Zhang, M. Cisse, Y. N. Dauphin, and D. Lopez-Paz, "mixup: Beyond empirical risk minimization," International Conference on Learning Representations (ICLR), 2018.

[148] S. Latif, R. Rana, S. Younis, J. Qadir, and J. Epps, "Cross corpus speech emotion classification-an effective transfer learning technique," arXiv preprint arXiv:1801.06353, 2018.

[149] X. J. Zhu, "Semi-supervised learning literature survey," University of Wisconsin-Madison Department of Computer Sciences, Tech. Rep., 2005.

[150] Z. Huang, M. Dong, Q. Mao, and Y. Zhan, "Speech emotion recognition using CNN," in Proceedings of the 22nd ACM international conference on Multimedia. ACM, 2014, pp. 801-804.

[151] J. Deng, X. Xu, Z. Zhang, S. Frühholz, and B. Schuller, "Semisupervised autoencoders for speech emotion recognition," IEEE/ACM Transactions on Audio, Speech, and Language Processing, vol. 26, no. 1, pp. 31-43, 2018.

[152] S. Parthasarathy, V. Rozgic, M. Sun, and C. Wang, "Improving emotion classification through variational inference of latent variables," in ICASSP 2019-2019 IEEE International Conference on Acoustics, Speech and Signal Processing (ICASSP). IEEE, 2019, pp. 7410-7414.

[153] J. Huang, Y. Li, J. Tao, Z. Lian, M. Niu, and J. Yi, "Speech emotion recognition using semi-supervised learning with ladder networks," in 2018 First Asian Conference on Affective Computing and Intelligent Interaction (ACII Asia). IEEE, 2018, pp. 1-5.

[154] S. Sahu, R. Gupta, and C. Espy-Wilson, "Modeling feature representations for affective speech using generative adversarial networks," IEEE Transactions on Affective Computing, 2020.

[155] — " "On enhancing speech emotion recognition using generative adversarial networks," INTERSPEECH, pp. 3693-3697, 2018.

[156] G. He, X. Liu, F. Fan, and J. You, "Image2Audio: Facilitating semisupervised audio emotion recognition with facial expression image," in Proceedings of the IEEE/CVF Conference on Computer Vision and Pattern Recognition Workshops, 2020, pp. 912-913.

[157] H. Zhao, Y. Xiao, and Z. Zhang, "Robust semisupervised generative adversarial networks for speech emotion recognition via distribution smoothness," IEEE Access, vol. 8, pp. 106 889-106 900, 2020.

[158] S. Sahu, R. Gupta, G. Sivaraman, W. AbdAlmageed, and C. EspyWilson, "Adversarial auto-encoders for speech based emotion recognition," INTERSPEECH, pp. 1243-1247, 2017.

[159] Y. Bengio, "Deep learning of representations for unsupervised and transfer learning," in Proceedings of ICML workshop on unsupervised and transfer learning, 2012, pp. 17-36.

[160] S. Thrun and L. Pratt, Learning to learn. Springer Science \& Business Media, 2012.

[161] J. Deng, Z. Zhang, F. Eyben, and B. Schuller, "Autoencoder-based unsupervised domain adaptation for speech emotion recognition," IEEE Signal Processing Letters, vol. 21, no. 9, pp. 1068-1072, 2014.

[162] J. Deng, R. Xia, Z. Zhang, Y. Liu, and B. Schuller, "Introducing shared-hidden-layer autoencoders for transfer learning and their application in acoustic emotion recognition," in 2014 IEEE International Conference on Acoustics, Speech and Signal Processing (ICASSP). IEEE, 2014, pp. 4818-4822.

[163] Y. Ganin, E. Ustinova, H. Ajakan, P. Germain, H. Larochelle, F. Laviolette, M. Marchand, and V. Lempitsky, "Domainadversarial training of neural networks," The journal of machine learning research, vol. 17, no. 1, pp. 2096-2030, 2016.

[164] Y. Xiao, H. Zhao, and T. Li, "Learning class-aligned and generalized domain-invariant representations for speech emotion recognition," IEEE Transactions on Emerging Topics in Computational Intelligence, vol. 4, no. 4, pp. 480-489, 2020.

[165] X. Cai, Z. Wu, K. Zhong, B. Su, D. Dai, and H. Meng, "Unsupervised cross-lingual speech emotion recognition using domain adversarial neural network," International Symposium on Chinese Spoken Language Processing, 2021. 
[166] H. Zhou and K. Chen, "Transferable positive/negative speech emotion recognition via class-wise adversarial domain adaptation," in ICASSP 2019-2019 IEEE International Conference on Acoustics, Speech and Signal Processing (ICASSP). IEEE, 2019, pp. 3732-3736.

[167] A. Batliner, S. Steidl, and E. Nöth, "Releasing a thoroughly annotated and processed spontaneous emotional database: the fau aibo emotion corpus," in Proc. of a satellite workshop of LREC, 2008, pp. 28-31.

[168] J. Gideon, M. McInnis, and E. M. Provost, "Improving crosscorpus speech emotion recognition with adversarial discriminative domain generalization (ADDoG)," IEEE Transactions on Affective Computing, 2019.

[169] S. Khorram, M. Jaiswal, J. Gideon, M. McInnis, and E.-M. Provost, "The priori emotion dataset: Linking mood to emotion detected in-the-wild," INTERSPEECH, pp. 1903-1907, 2018.

[170] R. Collobert and J. Weston, "A unified architecture for natural language processing: Deep neural networks with multitask learning," in Proceedings of the 25th international conference on Machine learning. ACM, 2008, pp. 160-167.

[171] L. Deng, G. Hinton, and B. Kingsbury, "New types of deep neural network learning for speech recognition and related applications: An overview," in 2013 IEEE International Conference on Acoustics, Speech and Signal Processing. IEEE, 2013, pp. 8599-8603.

[172] R. Girshick, "Fast R-CNN," in Proceedings of the IEEE international conference on computer vision, 2015, pp. 1440-1448.

[173] R. Caruana, "Learning to learn, chapter multitask learning," 1998.

[174] M.-R. Bouguelia, S. Pashami, and S. Nowaczyk, "Multi-task representation learning," in 30th Annual Workshop of the Swedish Artificial Intelligence Society SAIS 2017, May 15-16, 2017, Karlskrona, Sweden, no. 137. Linköping University Electronic Press, 2017, pp. 53-59.

[175] J. Kim, G. Englebienne, K. P. Truong, and V. Evers, "Towards speech emotion recognition" in the wild" using aggregated corpora and deep multi-task learning," in INTERSPEECH: Situated interaction, 2017, pp. 1113-1117.

[176] S. Parthasarathy and C. Busso, "Jointly predicting arousal, valence and dominance with multi-task learning," INTERSPEECH, 2017.

[177] B. Zhang, E. M. Provost, and G. Essl, "Cross-corpus acoustic emotion recognition with multi-task learning: Seeking common ground while preserving differences," IEEE Transactions on Affective Computing, no. 1, pp. 1-1, 2017.

[178] R. Raina, A. Battle, H. Lee, B. Packer, and A. Y. Ng, "Self-taught learning: transfer learning from unlabeled data," in Proceedings of the 24th international conference on Machine learning. ACM, 2007, pp. 759-766.

[179] L. Jing and Y. Tian, "Self-supervised visual feature learning with deep neural networks: A survey," IEEE Transactions on Pattern Analysis and Machine Intelligence, 2020.

[180] Z. Lan, M. Chen, S. Goodman, K. Gimpel, P. Sharma, and R. Soricut, “ALBERT: A Lite BERT for self-supervised learning of language representations," in International Conference on Learning Representations, 2019.

[181] A. Baevski, S. Schneider, and M. Auli, "VQ-WAV2VEC: Selfsupervised learning of discrete speech representations," in International Conference on Learning Representations, 2019.

[182] A. Shukla, K. Vougioukas, P. Ma, S. Petridis, and M. Pantic, "Visually guided self supervised learning of speech representations," in ICASSP 2020-2020 IEEE International Conference on Acoustics, Speech and Signal Processing (ICASSP). IEEE, 2020, pp. 6299-6303.

[183] S. Pascual, M. Ravanelli, J. Serrà, A. Bonafonte, and Y. Bengio, "Learning problem-agnostic speech representations from multiple self-supervised tasks," INTERSPEECH, pp. 161-165, 2019.

[184] A. Vaswani, N. Shazeer, N. Parmar, J. Uszkoreit, L. Jones, A. N. Gomez, Ł. Kaiser, and I. Polosukhin, "Attention is all you need," in Proceedings of the 31st International Conference on Neural Information Processing Systems, 2017, pp. 6000-6010.

[185] M. Macary, M. Tahon, Y. Estève, and A. Rousseau, "On the use of self-supervised pre-trained acoustic and linguistic features for continuous speech emotion recognition," in IEEE Spoken Language Technology Workshop, 2020.

[186] A. Khare, S. Parthasarathy, and S. Sundaram, "Self-supervised learning with cross-modal transformers for emotion recognition," IEEE Spoken Language Technology Workshop, 2020.

[187] J. Kober, J. A. Bagnell, and J. Peters, "Reinforcement learning in robotics: A survey," The International Journal of Robotics Research, vol. 32, no. 11, pp. 1238-1274, 2013.
[188] K. Arulkumaran, M. P. Deisenroth, M. Brundage, and A. A. Bharath, "Deep reinforcement learning: A brief survey," IEEE Signal Processing Magazine, vol. 34, no. 6, pp. 26-38, 2017.

[189] S. Latif, H. Cuayáhuitl, F. Pervez, F. Shamshad, H. S. Ali, and E. Cambria, "A survey on deep reinforcement learning for audiobased applications," arXiv preprint arXiv:2101.00240, 2021.

[190] H. Cuayáhuitl, S. Renals, O. Lemon, and H. Shimodaira, "Reinforcement learning of dialogue strategies with hierarchical abstract machines," in 2006 IEEE Spoken Language Technology Workshop. IEEE, 2006, pp. 182-185.

[191] J. Li, W. Monroe, A. Ritter, D. Jurafsky, M. Galley, and J. Gao, "Deep reinforcement learning for dialogue generation," in Proceedings of the 2016 Conference on Empirical Methods in Natural Language Processing, 2016, pp. 1192-1202.

[192] K.-F. Lee and S. Mahajan, "Corrective and reinforcement learning for speaker-independent continuous speech recognition," Computer Speech \& Language, vol. 4, no. 3, pp. 231-245, 1990.

[193] Y.-L. Shen, C.-Y. Huang, S.-S. Wang, Y. Tsao, H.-M. Wang, and T.-S. Chi, "Reinforcement learning based speech enhancement for robust speech recognition," in ICASSP 2019-2019 IEEE International Conference on Acoustics, Speech and Signal Processing (ICASSP). IEEE, 2019, pp. 6750-6754.

[194] J. Sangeetha and T. Jayasankar, "Emotion speech recognition based on adaptive fractional deep belief network and reinforcement learning," in Cognitive Informatics and Soft Computing. Springer, 2019, pp. 165-174.

[195] H. Li, B. Baucom, and P. Georgiou, "Unsupervised latent behavior manifold learning from acoustic features: Audio2behavior," in 2017 IEEE International Conference on Acoustics, Speech and Signal Processing (ICASSP). IEEE, 2017, pp. 5620-5624.

[196] F. Locatello, S. Bauer, M. Lucic, G. Raetsch, S. Gelly, B. Schölkopf, and $\mathrm{O}$. Bachem, "Challenging common assumptions in the unsupervised learning of disentangled representations," in international conference on machine learning. PMLR, 2019, pp. 4114-4124.

[197] T. Yang and C. E. Priebe, "The effect of model misspecification on semi-supervised classification," IEEE Transactions on Pattern Analysis and Machine Intelligence, vol. 33, no. 10, pp. 2093-2103, 2011.

[198] A. Singh, R. Nowak, and J. Zhu, "Unlabeled data: Now it helps, now it doesn't," Advances in neural information processing systems, vol. 21, pp. 1513-1520, 2008.

[199] Y.-F. Li and D.-M. Liang, "Safe semi-supervised learning: a brief introduction," Frontiers of Computer Science, vol. 13, no. 4, pp. 669676, 2019.

[200] A. Chatziagapi, G. Paraskevopoulos, D. Sgouropoulos, G. Pantazopoulos, M. Nikandrou, T. Giannakopoulos, A. Katsamanis, A. Potamianos, and S. Narayanan, "Data Augmentation Using GANs for Speech Emotion Recognition," in INTERSPEECH, 2019, pp. 171-175.

[201] F. Bao, M. Neumann, and N. T. Vu, "CycleGAN-based emotion style transfer as data augmentation for speech emotion recognition," Manuscript submitted for publication, pp. 35-37, 2019.

[202] Z. Gong, P. Zhong, and W. Hu, "Diversity in machine learning," IEEE Access, vol. 7, pp. 64 323-64 350, 2019.

[203] S. Latif, A. Qayyum, M. Usama, J. Qadir, A. Zwitter, and M. Shahzad, "Caveat emptor: the risks of using big data for human development," IEEE Technology and Society Magazine, vol. 38, no. 3, pp. 82-90, 2019.

[204] S. Yin, C. Liu, Z. Zhang, Y. Lin, D. Wang, J. Tejedor, T. F. Zheng, and $\mathrm{Y}$. Li, "Noisy training for deep neural networks in speech recognition," EURASIP Journal on Audio, Speech, and Music Processing, vol. 2015, no. 1, p. 2, 2015.

[205] F. Bu, Z. Chen, Q. Zhang, and X. Wang, "Incomplete big data clustering algorithm using feature selection and partial distance," in 2014 5th International Conference on Digital Home. IEEE, 2014, pp. 263-266.

[206] R. Wang and D. Tao, "Non-local auto-encoder with collaborative stabilization for image restoration," IEEE Transactions on Image Processing, vol. 25, no. 5, pp. 2117-2129, 2016.

[207] C. Breazeal and L. Aryananda, "Recognition of affective communicative intent in robot-directed speech," Autonomous robots, vol. 12, no. 1, pp. 83-104, 2002.

[208] N. Campbell, "Databases of emotional speech," in ISCA Tutorial and Research Workshop (ITRW) on Speech and Emotion, 2000.

[209] S. Asakawa, N. Minematsu, and K. Hirose, "Automatic recognition of connected vowels only using speaker-invariant representation of speech dynamics," in Eighth Annual Conference of the International Speech Communication Association, 2007. 
[210] Y. Lu, F. Lu, S. Sehgal, S. Gupta, J. Du, C. H. Tham, P. Green, and V. Wan, "Multitask learning in connectionist speech recognition," in Proceedings of the Australian International Conference on Speech Science and Technology, 2004.

[211] M. Jaiswal and E. M. Provost, "Privacy enhanced multimodal neural representations for emotion recognition," in Proceedings of the AAAI Conference on Artificial Intelligence, vol. 34, no. 05, 2020, pp. 7985-7993.

[212] S. Latif, J. Qadir, A. Qayyum, M. Usama, and S. Younis, "Speech technology for healthcare: Opportunities, challenges, and state of the art," IEEE Reviews in Biomedical Engineering, vol. 14, pp. 342-356, 2020

[213] M. A. Pathak, B. Raj, S. D. Rane, and P. Smaragdis, "Privacy-preserving speech processing: cryptographic and string-matching frameworks show promise," IEEE signal processing magazine, vol. 30, no. 2, pp. 62-74, 2013.

[214] B. M. L. Srivastava, A. Bellet, M. Tommasi, and E. Vincent, "Privacy-preserving adversarial representation learning in ASR: Reality or illusion?" Proc. INTERPSPEECH, pp. 3700-3704, 2019.

[215] R. Shokri and V. Shmatikov, "Privacy-preserving deep learning," in Proceedings of the 22nd ACM SIGSAC conference on computer and communications security. ACM, 2015, pp. 1310-1321.

[216] S. Latif, S. Khalifa, R. Rana, and R. Jurdak, "Federated learning for speech emotion recognition applications," in 2020 19th ACM/IEEE International Conference on Information Processing in Sensor Networks (IPSN). IEEE, 2020, pp. 341-342.

[217] S. Latif, R. Rana, and J. Qadir, "Adversarial machine learning and speech emotion recognition: Utilizing generative adversarial networks for robustness," arXiv preprint arXiv:1811.11402, 2018.

[218] I. J. Goodfellow, J. Shlens, and C. Szegedy, "Explaining and harnessing adversarial examples," International Conference on Learning Representations (ICLR), 2015.

[219] N. Papernot, P. McDaniel, S. Jha, M. Fredrikson, Z. B. Celik, and A. Swami, "The limitations of deep learning in adversarial settings," in 2016 IEEE European Symposium on Security and Privacy (EuroSEP). IEEE, 2016, pp. 372-387.

[220] S.-M. Moosavi-Dezfooli, A. Fawzi, and P. Frossard, "Deepfool: a simple and accurate method to fool deep neural networks," in Proceedings of the IEEE Conference on Computer Vision and Pattern Recognition, 2016, pp. 2574-2582.

[221] Z. Ren, A. Baird, J. Han, Z. Zhang, and B. Schuller, "Generating and protecting against adversarial attacks for deep speechbased emotion recognition models," in ICASSP 2020-2020 IEEE International Conference on Acoustics, Speech and Signal Processing (ICASSP). IEEE, 2020, pp. 7184-7188.

[222] N. Akhtar and A. Mian, "Threat of adversarial attacks on deep learning in computer vision: A survey," IEEE Access, vol. 6, pp. 14 410-14 430, 2018.

[223] J. Chen, J. Konrad, and P. Ishwar, "A cyclically-trained adversarial network for invariant representation learning," in Proceedings of the IEEE/CVF Conference on Computer Vision and Pattern Recognition Workshops, 2020, pp. 782-783.

[224] X. Yuan, P. He, Q. Zhu, and X. Li, "Adversarial examples: Attacks and defenses for deep learning," IEEE Transactions on Neural Networks and Learning Systems, vol. 30, no. 9, pp. 2805-2824, 2019.

[225] M. Sarma, P. Ghahremani, D. Povey, N. K. Goel, K. K. Sarma, and N. Dehak, "Improving emotion identification using phone posteriors in raw speech waveform based DNN," INTERSPEECH, pp. 3925-3929, 2019.

[226] P. J. Werbos, "Backpropagation through time: what it does and how to do it," Proceedings of the IEEE, vol. 78, no. 10, pp. 15501560,1990

[227] S. Karita, N. Chen, T. Hayashi, T. Hori, H. Inaguma, Z. Jiang, M. Someki, N. E. Y. Soplin, R. Yamamoto, X. Wang et al., "A comparative study on transformer vs RNN in speech applications," in 2019 IEEE Automatic Speech Recognition and Understanding Workshop (ASRU). IEEE, 2019, pp. 449-456.

[228] A. Zeyer, P. Bahar, K. Irie, R. Schlüter, and H. Ney, "A comparison of transformer and LSTM encoder decoder models for ASR," in 2019 IEEE Automatic Speech Recognition and Understanding Workshop (ASRU). IEEE, 2019, pp. 8-15.

[229] J. Li, Y. Wu, Y. Gaur, C. Wang, R. Zhao, and S. Liu, "On the comparison of popular end-to-end models for large scale speech recognition," INTERSPEECH, pp. 1-5, 2020.

[230] N. Li, S. Liu, Y. Liu, S. Zhao, and M. Liu, "Neural speech synthesis with transformer network," in Proceedings of the AAAI Conference on Artificial Intelligence, vol. 33, no. 01, 2019, pp. 6706-6713.
[231] D. Lim, W. Jang, O. Gyeonghwan, H. Park, B. Kim, and J. Yoon, "JDI-T: Jointly trained duration informed transformer for textto-speech without explicit alignment," INTERSPEECH, pp. 4004$4008,2020$.

[232] Z. Zhang, J. Han, J. Deng, X. Xu, F. Ringeval, and B. Schuller, "Leveraging unlabeled data for emotion recognition with enhanced collaborative semi-supervised learning," IEEE Access, vol. 6, pp. 22 196-22 209, 2018.

[233] Z. Huang, M. Dong, Q. Mao, and Y. Zhan, "Speech emotion recognition using CNN," in Proceedings of the 22Nd ACM International Conference on Multimedia, 2014.

[234] G. Pironkov, S. Dupont, and T. Dutoit, "Multi-task learning for speech recognition: an overview," in ESANN, 2016.

[235] V. Thomas, E. Bengio, W. Fedus, J. Pondard, P. Beaudoin, H. Larochelle, J. Pineau, D. Precup, and Y. Bengio, "Disentangling the independently controllable factors of variation by interacting with the world," Learning Disentangling Representations Workshop (NeurIPS), 2017.

[236] C. Gelada, S. Kumar, J. Buckman, O. Nachum, and M. G. Bellemare, "DeepMDP: Learning continuous latent space models for representation learning," in International Conference on Machine Learning. PMLR, 2019, pp. 2170-2179.

[237] H. Van Seijen, M. Fatemi, J. Romoff, R. Laroche, T. Barnes, and J. Tsang, "Hybrid reward architecture for reinforcement learning," in Advances in Neural Information Processing Systems, 2017, pp. 5392-5402.

[238] M. Jaderberg, V. Mnih, W. M. Czarnecki, T. Schaul, J. Z. Leibo, D. Silver, and K. Kavukcuoglu, "Reinforcement learning with unsupervised auxiliary tasks," International Conference on Learning Representations, 2017.

[239] T. Zhang, M. Huang, and L. Zhao, "Learning structured representation for text classification via reinforcement learning," in Thirty-Second AAAI Conference on Artificial Intelligence, 2018.

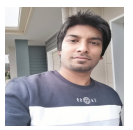

Siddique Latif is completing $\mathrm{PhD}$ at the University of southern Queensland (USQ), Australia and Distributed Sensing Systems research group, Data61-CSIRO. His PhD work focuses on representation learning, using unlabelled, weakly-labelled, partially-labelled multi-modal data.

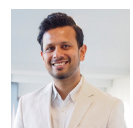

Rajib Rana is an experimental computer scientist, Advance Queensland Research Fellow and a Senior Lecturer in the University of Southern Queensland. He is also the Director of loT Health research program at the University of Southern Queensland. His current research focus is on Unsupervised Representation Learning.

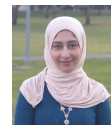

Sara Khalifa is currently a senior research scientist at the Distributed Sensing Systems research group, Data61-CSIRO. Her research interests include Internet of Things, smart wearables, energy harvesting, and pattern recognition. She obtained a $\mathrm{PhD}$ in Computer Science and Engineering from UNSW (Sydney, Australia).

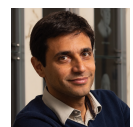

Raja Jurdak is a Professor of Distributed Systems and Chair in Applied Data Sciences at Queensland University of Technology, and Director of the Trusted Networks Lab. His research interests include trust, mobility and energyefficiency in networks.

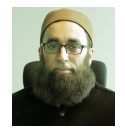

Junaid Qadir (SM'14) is a Professor at the Qatar University in Doha, Qatar, and the Information Technology University (ITU) of Punjab in Lahore, Pakistan. He directs the IHSAN Research Lab. His primary research interests are in the areas of computer systems and networking, and applied machine learning.

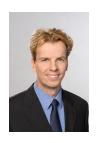

Björn W. Schuller (M'05-SM'15-F'18) is Professor of Artificial Intelligence in the Department of Computing at the Imperial College London/UK, where he heads GLAM - the Group on Language, Audio \& Music, Full Professor and head of the ZD.B Chair of Embedded Intelligence for Health Care and Wellbeing at the University of Augsburg/Germany, and CEO of audEERING. 